\title{
Quantifying errors due to frequency changes and target location uncertainty for radar refractivity retrievals
}

Article

Published Version

Nicol, J. C., Illingworth, A. J., Darlington, T. and Kitchen, M. (2013) Quantifying errors due to frequency changes and target location uncertainty for radar refractivity retrievals. Journal of Atmospheric and Oceanic Technology, 30 (9). pp. 2006-2024. ISSN 0739-0572 doi: https://doi.org/10.1175/JTECH-D-1200118.1 Available at https://centaur.reading.ac.uk/36317/

It is advisable to refer to the publisher's version if you intend to cite from the work. See Guidance on citing.

To link to this article DOI: http://dx.doi.org/10.1175/JTECH-D-12-00118.1

Publisher: American Meteorological Society

All outputs in CentAUR are protected by Intellectual Property Rights law, including copyright law. Copyright and IPR is retained by the creators or other copyright holders. Terms and conditions for use of this material are defined in the End User Agreement.

www.reading.ac.uk/centaur 
Central Archive at the University of Reading

Reading's research outputs online 


\title{
Quantifying Errors due to Frequency Changes and Target Location Uncertainty for Radar Refractivity Retrievals
}

\author{
J. C. NICOL \\ National Centre for Atmospheric Science, University of Reading, Reading, United Kingdom \\ A. J. ILLINGWORTH \\ University of Reading, Reading, United Kingdom \\ T. DARLINGTON AND M. KITCHEN \\ Met Office, Exeter, United Kingdom
}

(Manuscript received 15 May 2012, in final form 12 April 2013)

\begin{abstract}
Radar refractivity retrievals can capture near-surface humidity changes, but noisy phase changes of the ground clutter returns limit the accuracy for both klystron- and magnetron-based systems. Observations with a C-band $(5.6 \mathrm{~cm})$ magnetron weather radar indicate that the correction for phase changes introduced by local oscillator frequency changes leads to refractivity errors no larger than $0.25 \mathrm{~N}$ units: equivalent to a relative humidity change of only $0.25 \%$ at $20^{\circ} \mathrm{C}$. Requested stable local oscillator (STALO) frequency changes were accurate to $0.002 \mathrm{ppm}$ based on laboratory measurements. More serious are the random phase change errors introduced when targets are not at the range-gate center and there are changes in the transmitter frequency $\left(\Delta f_{\mathrm{Tx}}\right)$ or the refractivity $(\Delta N)$. Observations at $\mathrm{C}$ band with a $2-\mu \mathrm{s}$ pulse show an additional $66^{\circ}$ of phase change noise for a $\Delta f_{\mathrm{Tx}}$ of $190 \mathrm{kHz}(34 \mathrm{ppm})$; this allows the effect due to $\Delta N$ to be predicted. Even at $\mathrm{S}$ band with klystron transmitters, significant phase change noise should occur when a large $\Delta N$ develops relative to the reference period [e.g., $\sim 55^{\circ}$ when $\Delta N=60$ for the Next Generation Weather Radar (NEXRAD) radars]. At shorter wavelengths (e.g., $\mathrm{C}$ and $\mathrm{X}$ band) and with magnetron transmitters in particular, refractivity retrievals relative to an earlier reference period are even more difficult, and operational retrievals may be restricted to changes over shorter (e.g., hourly) periods of time. Target location errors can be reduced by using a shorter pulse or identified by a new technique making alternate measurements at two closely spaced frequencies, which could even be achieved with a dual-pulse repetition frequency (PRF) operation of a magnetron transmitter.
\end{abstract}

\section{Introduction}

Radar refractivity retrieval is a relatively new application of weather radar measurements, originally proposed by Fabry et al. (1997). During summer, refractivity changes are typically dominated by near-surface humidity changes. It is anticipated that retrievals will provide valuable insights into the dynamic variability of nearsurface water vapor and may be a valuable new data source for assimilation into numerical weather prediction

Corresponding author address: Dr. John Nicol, National Centre for Atmospheric Science, Dept. of Meteorology, University of Reading, Whiteknights, P.O. Box 243, Reading RG6 6BB, United Kingdom.

E-mail: j.c.nicol@reading.ac.uk models, particularly with respect to the initiation of convection. Radar refractivity retrieval essentially uses the phase change between two different plan position indicator (PPI) radar scans from stationary targets (ground clutter). The ground clutter field under standard propagation conditions is usually limited to a range of $50 \mathrm{~km}$ or so. The phase change for a particular target (targ) may be expressed as

$$
\Delta \phi_{\mathrm{targ}}=-\frac{4 \pi f_{\mathrm{Tx}} r_{\operatorname{targ}}}{c} \Delta n .
$$

Here, $f_{\text {Tx }}$ is the transmitter frequency, $r_{\text {targ }}$ is the target distance, and $\Delta n$ is the mean change in the refractive index between the two times along the path between the 
radar and the target. This expression differs in sign convention from (2) in Fabry et al. (1997) but conforms with the standard relationship between Doppler velocity and phase (Doviak and Zrnic 2006) as discussed in the appendix. Considering phase change differences between two targets ( $\mathrm{A}$ and $\mathrm{B}$ ), separated in range along a given azimuth, we have

$$
\Delta \phi_{B}-\Delta \phi_{A}=-\frac{4 \pi f_{\mathrm{Tx}}}{c} \Delta n\left(r_{B}-r_{A}\right) .
$$

Henceforth, $n, \Delta n$, and refractivity changes $\Delta N[N=$ $(n-1) \times 10^{6}$ ] denote the mean values between two targets or between the radar and the target when a single target is considered. Rearranging (2) and in terms of refractivity changes gives

$$
\Delta N=-\frac{c}{4 \pi f_{\mathrm{Tx}}} 10^{6} \frac{\Delta \phi_{B}-\Delta \phi_{A}}{r_{B}-r_{A}} .
$$

Phase change differences $\left(\Delta \phi_{B}-\Delta \phi_{A}\right)$ are typically calculated by "pulse-pair" processing (e.g., Skolnik 1990, p. 23.15), although changes are estimated between adjacent range gates rather than successive pulses as for Doppler velocity. At S-, C-, and X-band wavelengths, the sensitivity of the phase change differences to refractivity changes is approximately $7^{\circ}, 13^{\circ}$, and $23^{\circ} \mathrm{km}^{-1}$, respectively, for $\Delta N=1$. Fabry et al. (1997) describe how changes may be determined relative to a reference period lasting an hour or so, identified weeks or even months previously, when the refractivity field had a known value and was almost homogeneous over the clutter field; the reference phase field was obtained by averaging the phases over scans during such a period. This would then allow fields of absolute refractivity changes to be derived from subsequent scans. Clearly, difficulties in detecting large values of $\Delta N$ will increasingly occur at C-band and even more so at X-band wavelengths due to aliasing when $\left(\Delta \phi_{B}-\Delta \phi_{A}\right)$ exceeds $\pm 180^{\circ}$ over the distance $\left(r_{B}-r_{A}\right)$. These difficulties are accentuated because large random phase change errors are usually present, such as those due to target motion. At shorter wavelengths, phase change noise is greater, and finding suitably quiescent reference periods becomes more difficult due to the increased sensitivity to refractivity changes.

Refractivity retrievals have previously been demonstrated for radars with klystron transmitters both for typical weather radars with parabolic reflector antennas (e.g., Fabry et al. 1997; Fabry 2004, 2006; Weckwerth et al. 2005; Roberts et al. 2008) and for phased array radars (Cheong et al. 2008). Klystrons are very stable in terms of transmitter frequency, but magnetron transmitters, which are widely used outside the United States, are prone to drift. It is perhaps due to the incomplete understanding of the subtle effects of these frequency drifts that refractivity retrievals have had only limited application for magnetron-based radars.

The magnetron transmitter frequency changes primarily with the ambient temperature and changes in the duty cycle related to the pulse repetition frequency (PRF) or pulse duration (Skolnik 1990, p. 4.8). Junyent et al. (2010) examined the transmitter frequency of the X-band Collaborative Adaptive Sensing of the Atmosphere (CASA) radars and found an anode temperature dependence of $-166 \mathrm{kHz}^{\circ} \mathrm{C}^{-1}$ and changes of about $-550 \mathrm{kHz}(\sim 60 \mathrm{ppm})$ per $0.025 \%$ duty cycle increment. Their Fig. 10 shows a huge decrease of over $4 \mathrm{MHz}$ (>400 ppm) in $30 \mathrm{~min}$, presumably after start up.

Parent du Chatelet et al. (2007) were the first to suggest that the phase changes from stationary targets are primarily related to changes in the local oscillator rather than the transmitter frequency. The phase changes due to both transmitter and local oscillator frequency changes were formulated as a function of time (delay) by Parent du Chatelet and Boudjabi (2008), Junyent et al. (2009), and Parent du Chatelet et al. (2012, hereafter PC2012). PC2012 identified three terms in their expression for phase changes: the "local oscillator" term, the "mismatch" term, and the "refractivity" term. They proposed that corrections for the local oscillator term could be applied if the local oscillator frequency is precisely known. In the context of refractivity retrievals using a single target over a 3-km path from the radar, they concluded that the mismatch term resulted in additive refractivity errors that could generally be neglected. However, refractivity retrievals are typically based on the estimation of phase change gradients using the target pairs expressed in (3), often between adjacent range gates (e.g., Cheong et al. 2008) to minimize aliasing; in these cases we will show that phase change noise arising from the mismatch term is significant.

In this paper, we present an expression for the measured phase change in terms of range (rather than sampling and propagation delays) when there are changes in both the transmitter and local oscillator (LO) frequencies (originally given by Nicol et al. 2008). This expression makes it clear that for the mismatch term the source of phase change error is, in fact, related to target location uncertainty. There is also a second term involving target location uncertainty whereby refractivity changes lead to phase change errors; this term affects both magnetron and klystron radar systems. Far from being negligible, these terms and the resulting phase change noise due to target location uncertainty are very important for refractivity retrievals. 
The fundamental expression for the measured phase change as a function of range is presented and interpreted in section 2 (a full derivation is provided in the appendix). In section 3, we describe the C-band radar system used in this study and the in situ measurements from a surface station used for validation. Refractivity retrievals using a single target close to the radar are used to demonstrate the necessary correction for LO frequency changes. In section 4 , we quantify the refractivity estimation errors due to the correction for LO frequency changes. This has been achieved based on laboratory measurements and by identifying phase-correlated returns (where a single target contributes significant returns over adjacent range gates), from which we confirm that the down conversion from the intermediate frequency (IF; adjusted on a scan-by-scan basis) is achieved with a suitably high degree of accuracy $(<0.2 \mathrm{ppm})$. In section 5 , the observed increase in phase change noise associated with transmitter frequency changes is used to quantify target location uncertainty; this implies that when there is a large change in refractivity, the target location uncertainty can lead to significant phase change noise and degrade refractivity retrievals not only for magnetrons but also for radars using klystrons. The mitigation of target location uncertainty is the subject of section 6 , where the advantages of short pulses are discussed and a technique to ascertain the precise location of the target within the range gate is proposed. Finally the conclusions are summarized in section 7 .

\section{Influence of frequency changes on phase measurements in terms of target range}

In this section, we describe and interpret the effects of frequency changes on phase measurements as a function of target range. We define the LO frequency as the sum of the local oscillator frequencies, whether this combines the stable local oscillator (STALO) and the coherent oscillator (COHO) for an analog IF or the STALO and the numerically controlled oscillator (NCO) for a digital IF. The STALO is used to mix radio frequencies down to IF and the final stage (COHO or NCO) mixes this signal down to baseband. In magnetron systems, automatic frequency control (AFC) is implemented by adjusting the LO frequency to follow the transmitter frequency typically in a near-continuous fashion or in discrete steps for analog and digital IF, respectively. The radar considered in this work (along with the other radars in the operational U.K. network) is perhaps unique in that the AFC is implemented by adjusting both the STALO (from time to time) and the NCO (prior to each and every scan) to provide very high precision frequency down conversion.
We shall assume that a single-point clutter target dominates returns at each range gate; Hubbert et al. (2009) found that modeling clutter returns in this manner best replicated measurements of clutter phase alignment from the Denver, Colorado, Front Range Airport (KFTG) Next Generation Weather Radar (NEXRAD). The intrinsic phase of a clutter target not only depends on the range and structure of the target but also on how the target is illuminated by the radar beam. Changes in the vertical refractivity gradient affect the propagation of the radar waves and consequently the intrinsic phase may deviate from its value under standard propagation conditions. These effects will be the greatest during periods of anomalous propagation and at further ranges (Fabry 2004) and may be important for refractivity retrievals. However, the equivalent changes in the effective range of the target are likely to be negligible considering the effects of frequency changes.

\section{The effect of frequency changes on the return phases from a stationary target}

The phase change $\left(\Delta \phi_{\text {gate }}\right)$ w.r.t. time of returns from a stationary target sampled at a range gate centered at a given range $\left(r_{\text {gate }}\right)$ from the radar, corresponding to $\mathrm{LO}$ and transmitter frequency changes $\left(\Delta f_{\mathrm{LO}}\right.$ and $\Delta f_{\mathrm{Tx}}$, respectively) and refractivity changes $(\Delta n)$, may be expressed in radians as

$$
\begin{aligned}
\Delta \phi_{\text {gate }}= & -\frac{4 \pi}{c}\left[r_{\text {gate }} \Delta f_{\mathrm{LO}}+\delta_{\text {gate }} \Delta f_{\mathrm{Tx}}\right. \\
& \left.+\left(r_{\text {gate }}+\delta_{\text {gate }}\right) f_{\mathrm{Tx}} \Delta n\right],
\end{aligned}
$$

where $\delta_{\text {gate }}=r_{\text {targ }}-r_{\text {gate }}$ is the distance of the target from the center of the range gate. The derivation is given in the appendix. The range-gate center considered here is the equivalent range-gate center in a vacuum $(n=1)$ and is defined by the external clock that triggers the analog-to-digital (A-D) converter (ADC). Equation (4) is essentially equivalent to (8) in PC2012, though expressed in terms of range rather than time delays and differing in some subtle yet important definitions. The first term is the local oscillator term of PC2012, and the second term is their mismatch term, which they suggested could generally be neglected; in section 5, we demonstrate that this term can be important and is due to target location uncertainty $\left(\delta_{\text {gate }}\right)$. The presence and significance of $\delta_{\text {gate }}$ in the third refractivity term was not identified in PC2012. Target location uncertainty can lead to significant phase change noise due to both transmitter frequency and refractivity changes.

The origins of the first two terms in (4) may be visualized in Fig. 1 where we have assumed that $\Delta n=0$ and both the transmitter $\left(T_{0}\right)$ and $\mathrm{LO}$ waveforms have zero 


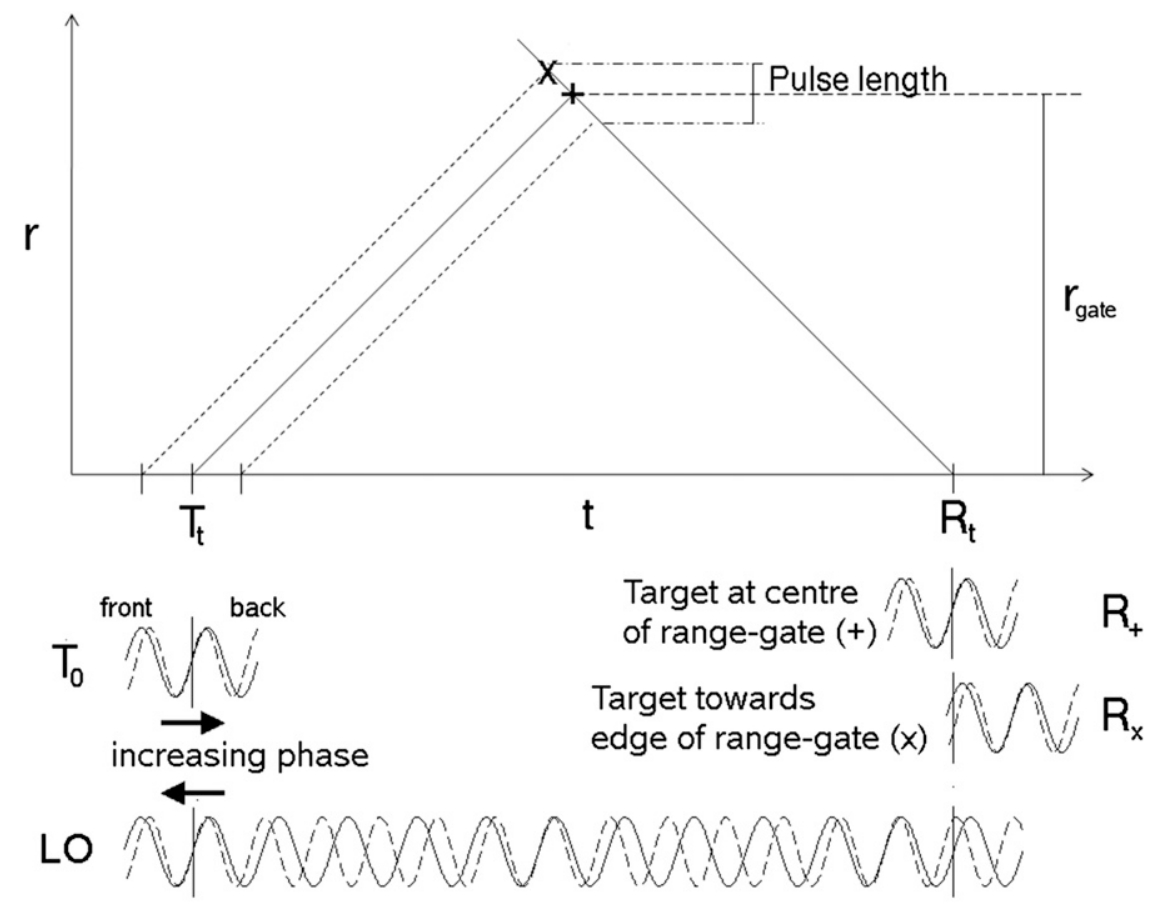

FIG. 1. Schematic diagram of distance vs time, showing the path of the radar pulse from transmission at time $T_{t}$ to reception at time $R_{t}$. The transmitter $\left(T_{0}\right)$ and $\mathrm{LO}$ waveforms are both depicted at an earlier time (solid sine waves) and a later time (dashed sine waves) representing a positive change in frequency. The phase change due to LO frequency changes is proportional to the time $\left(T_{t}-R_{t}\right)$. From the transmitted pulse, the received signal can involve returns from targets at any distance from the radar $(r)$ within the range gate (pulse length) centered at distance $r_{\text {gate }}$. When a target lies at the center of the range gate (symbol + at $r_{\text {gate }}$ ), no phase change will occur because of the transmitter frequency changes. For targets located away from the range-gate center (symbol $\mathrm{x}$ ), the phase change due to transmitter frequency changes increases with the distance from the center of the range gate essentially caused by the different propagation time. The total phase change relates to the sum of those due to transmitter and LO frequency changes.

phase at a time corresponding to the transmission of the center of the pulse $\left(T_{t}\right)$. In this work, we use the standard convention of positive Doppler away from the radar, which implies that the phase of the transmitter waveform increases with time so that it increases toward the back or trailing edge of the pulse. If we consider that the phase of the received signal is added to the phase of the LO signal, then the phase of the LO waveform effectively decreases with time. The returned signal is mixed with the LO signal and sampled at a time $R_{t}$, defined by an external clock that triggers the A-D converter. The phase of the signal at baseband is then simply the sum of the returned and LO phases. At time $R_{t}$, the receiver will receive echoes from all targets along the sloping line provided they are illuminated by the transmit pulse. In the figure, we distinguish between a target at range "+," which is in the center of the range gate and has return signal $R_{+}$, and a target at range "x," which is a distance $\delta_{\text {gate }}$ from the center of the range gate with a return signal $R_{\mathrm{X}}$. In Fig. 1, the waveforms for the first pulse are represented by solid sine waves while the dashed sine waves are for a pulse at a subsequent time when there are positive frequency shifts, $\Delta f_{\mathrm{Tx}}$ and $\Delta f_{\mathrm{LO}}$. The return phase $\phi_{\text {gate }}$ is given by the sum of the LO phase and the $R_{+}$or $R_{\mathrm{x}}$ phases, and the value of $\Delta \phi_{\text {gate }}$ in (4) is the difference in phase between the value for the later pulse (dashed sine waves) and the first pulse (solid sine waves), each sampled at the time $R_{t}$ relative to the transmitted pulse, indicated by the vertical lines in Fig. 1.

The first and second terms in (4) give rise to spurious phase changes and are typically confined to magnetrons. The first (LO) term is shown in Fig. 1, where a positive $\Delta f_{\mathrm{LO}}$ leads to a decrease in $\Delta \phi$. In the diagram, there is an apparent phase change of about $-785^{\circ}$ at this range gate (observed as $-65^{\circ}$ after aliasing, assuming that phase changes range from $-180^{\circ}$ to $\left.180^{\circ}\right)$. This is completely 
independent of propagation effects and target location. The LO contribution to $\Delta \phi$ is proportional to the sampling range and can become very large for ranges of tens of kilometers (e.g., $135^{\circ}$ every $10 \mathrm{~km}$ for a $1 \mathrm{ppm}$ change in the local oscillator frequency).

The second (Tx target location error) term arises because of the difference between the propagation delay and the sampling delay and is therefore proportional to the target range relative to the range-gate center $\left(\delta_{\text {gate }}\right)$ when there is a change in transmit frequency, as shown by the difference in the dashed and solid sine wave $R_{x}$. In Fig. 1, the target is located farther away from the rangegate center so the return corresponds to the leading edge of the transmitted pulse, so a positive $\Delta f_{\mathrm{Tx}}$ again results in a decrease in $\Delta \phi$. For a target at the range-gate center, $\Delta \phi$ is unaffected by $\Delta f_{\mathrm{Tx}}$ as indicated by the returned signal $R_{+}$.

The final (refractivity) term contains the desired information on refractivity and depends on the target range from the radar $\left(r_{\text {gate }}+\delta_{\text {gate }}\right)$ and the mean change in the refractive index. In practice, it is typically assumed that the target is in the center of the range gate $\left(\delta_{\text {gate }}=\right.$ 0 ), in which case it corresponds to (2) in Fabry et al. (1997) except for the sign convention. For a positive $\Delta n$, the transmitted pulse travels more slowly and, as the returned signal is sampled after a fixed delay $\left(R_{t}-T_{t}\right)$, the sample corresponds to an earlier part of the transmitted waveform or once again a decrease in $\Delta \phi$. The situation is equivalent to the two solid waves $R_{+}$and $R_{x}$, but in this case the time delay is caused by the refractivity increase rather than the extra path $\left(2 \delta_{\text {gate }}\right)$. The final component of the refractivity term (proportional to $\delta_{\text {gate }} \Delta n$ ) may be identified as a refractivity target location error, a new term analogous to the $\mathrm{Tx}$ target location error. This error can also be significant for klystron systems (Nicol and Illingworth 2013). We shall later quantify the magnitude of these terms in section 5 .

\section{Demonstrating the effect of LO frequency changes using retrievals from a single target}

In this section, we shall demonstrate radar refractivity retrieval using a single target located close to the radar in comparison with surface observations. We initially describe the C-band magnetron radar systems operated as part of the U.K. operational weather radar network and the in situ surface observations used for validation. The analysis of data from one of these radars, located at Cobbacombe in southwest England, confirms that the continuously adjusted (from scan to scan) LO frequency can introduce large errors into estimates of $\Delta N$ but that a simple correction may be easily applied.

\section{a. Radar system specifications and signal processing}

Currently, all the radars of the U.K. operational network are C-band (5.6- $\mathrm{cm}$ wavelength) radars with magnetron transmitters. The Met Office has recently developed its own digital-IF Doppler radar processing system using commercial off-the-shelf hardware and inhouse software. This system uses a 100-MHz 14-bit ADC to capture IF samples of both the received signal and the transmitter pulse and controls both the STALO (Pascall OCXO) and NCO in the down conversion. Samples of the received signal at baseband are digitally filtered and subsampled to provide the in-phase and quadrature components with a range resolution matching the pulse duration. The finite impulse response (FIR) filters introduce a delay, though they do not affect the phase because of their linear response. Because of the digital nature of the NCO, the frequency used for baseband down conversion can be controlled very accurately. Thus, with good frequency estimation from samples of the transmitted pulse at IF, it is possible to convert to baseband with an accuracy limited only by the frequency precision of the oscillator used to clock the field programmable gate array (FPGA) and ADC. The specified precision of the oscillator used for the FPGA and ADC $(\sim 20 \mathrm{ppm})$ implies that the NCO signal $(\sim 30 \mathrm{MHz})$ should be accurate to about $600 \mathrm{~Hz}$ or about $0.1 \mathrm{ppm}$ relative to the transmitter frequency.

The transmitter frequency is constantly monitored and recorded during radar operation. This is achieved by sampling a portion of the transmitter pulse mixed to IF and estimating the frequency from a regression algorithm. Initial IF estimation is carried out by the interpolation of the peak of a fast Fourier transform (FFT) of the transmitter burst samples. This is used to initialize a minimization procedure to improve the accuracy of the estimate by fitting the sampled data to the idealized transmitter pulse:

$$
s(t)=A \cos (2 \pi f t+\phi)+b,
$$

where $b$ allows for DC offsets in the ADC. The estimated transmitter frequency is then the sum of the estimated IF and the digitally requested STALO frequency. Generally, the STALO frequency is held constant, and the NCO is adjusted to provide high-precision AFC (Darlington 2010), which is equivalent to AFC in analog IF systems where LO frequency adjustments are almost continuously applied. When the deviation of the estimated frequency from the nominal IF $(30 \mathrm{MHz})$ becomes large $(>100 \mathrm{kHz})$, the STALO is reset to provide an IF close to $30 \mathrm{MHz}$. Immediately prior to the start of each PPI scan, the NCO frequency is chosen to match 

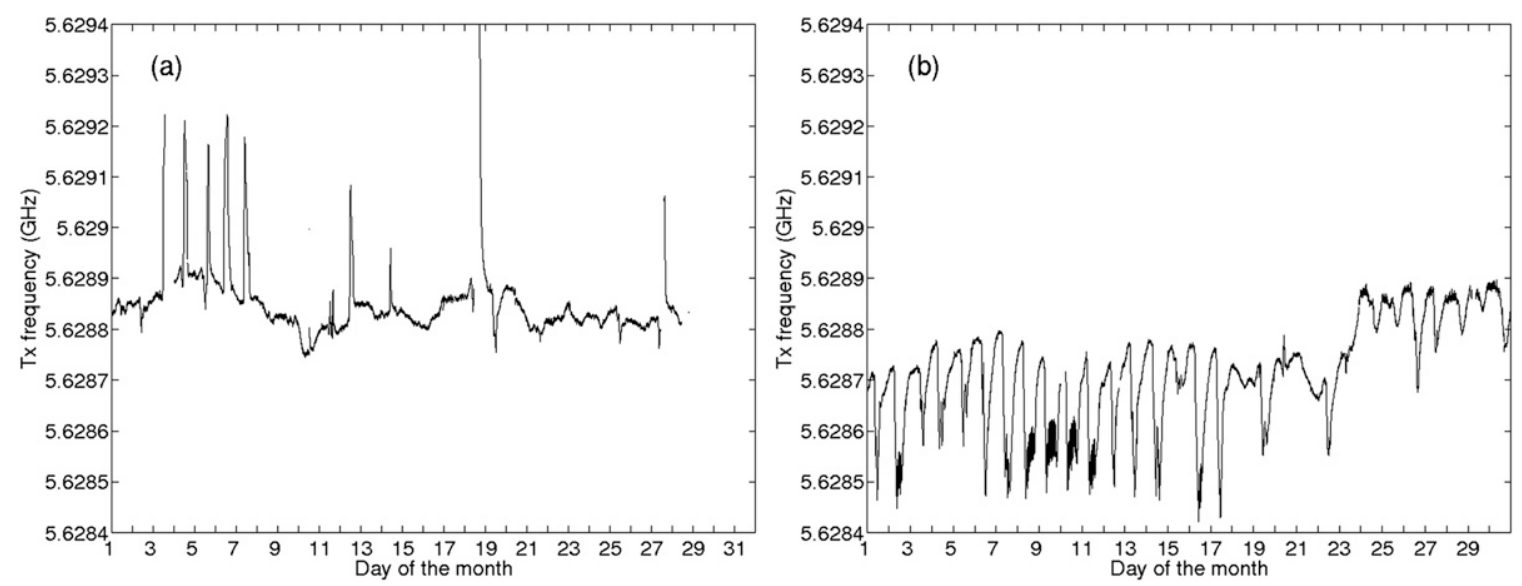

FIG. 2. Measured transmitter frequency for the radar at Cobbacombe during (a) March 2008 and (b) June 2008.

the estimated IF, allowing for any changes requested from the STALO. The transmitter frequency is then recorded for each scan and is, by definition, equal to the LO frequency (i.e., the sum of the STALO and NCO frequencies). In contrast, it is of course possible to only change the LO frequency in relatively large discrete steps. Figure 10 in Junyent et al. (2010) implies that these steps are of $250 \mathrm{kHz}$ for the $\mathrm{X}$-band CASA radars. We will show in section 4a that, for the Met Office C-band radar, both the IF measurements and the requested STALO and NCO frequency changes are exceptionally accurate.

The inferred changes in transmitter frequency for the months of March and June 2008 are plotted in Fig. 2 and show that in the month of June the frequency can fall by up to $300 \mathrm{kHz}$ (over $50 \mathrm{ppm}$ ) on warm sunny days. In March, the trace has sudden sharp increases in the frequency of $300 \mathrm{kHz}$ coinciding with site visits by engineers, and it is likely that these changes are dominated by changes in power usage at the site rather than temperature changes. Data were obtained from PPI scans at the lowest operational elevation $\left(0^{\circ}\right)$ every $5 \mathrm{~min}$, using a $2-\mu$ s pulse. The radar specifications and operating parameters are given in Table 1.

\section{b. In situ surface station measurements}

Measurements from the Met Office surface station at Dunkeswell, some $20-\mathrm{km}$ southeast of the radar, have been used for validation. Refractivity was derived from observations made every minute of temperature $T(\mathrm{~K})$, pressure $p(\mathrm{hPa})$, and relative humidity [converted to partial water vapor pressure $e(\mathrm{hPa})$ ], using the relation of Bean and Dutton (1968):

$$
N=77.6 \frac{p}{T}+3.73 \times 10^{5} \frac{e}{T^{2}} .
$$

Figure 3 shows the refractivity time series for March and June 2008. The instantaneous 1-min values have been smoothed over $11 \mathrm{~min}$ to reduce the high-frequency fluctuations of up $3 \mathrm{~N}$ units during summer associated with small-scale structures (Bartholomew 2012).

\section{c. Influence of local oscillator frequency changes}

We have seen in section 2 that phase changes due to LO frequency changes are predictable and increase linearly with range and $\Delta f_{\mathrm{LO}}$. Assuming the target is in the center of the range gate $\left(\delta_{\text {gate }}=0\right)$, then because $f_{\mathrm{LO}} \approx f_{\mathrm{Tx}}$, (4) then becomes

$$
\begin{aligned}
\Delta \phi_{\text {gate }} & =-\frac{4 \pi r_{\text {gate }}}{c}\left(\Delta f_{\mathrm{LO}}+f_{\mathrm{Tx}} \Delta n\right) \\
& \approx-\frac{4 \pi r_{\text {gate }} f_{\mathrm{Tx}} 10^{-6}}{c}\left(\Delta F_{\mathrm{LO}}+\Delta N\right) .
\end{aligned}
$$

The difference between $f_{\mathrm{LO}}$ and $f_{\mathrm{Tx}}$ must typically be much less than one part per thousand to ensure that the received signal falls within the radar bandwidth. It is apparent from (7) that a fractional LO frequency change in ppm, $\Delta F_{\mathrm{LO}}=\left(\Delta f_{\mathrm{LO}} / f_{\mathrm{LO}}\right) 10^{6}$, has the same effect as an equivalent refractivity change in ppm $(\Delta N)$. If no correction is made for LO frequency changes, estimates of the refractivity change will have an additive bias equal to $\Delta F_{\mathrm{LO}}$. However, if LO frequency changes are recorded,

TABLE 1. Technical specifications of U.K. weather radars and operational parameters for the low elevation scan.

\begin{tabular}{ll}
\hline \hline Frequency & $5.6 \mathrm{GHz}$ \\
Wavelength & $5.4 \mathrm{~cm}$ \\
PRF & $300 \mathrm{~Hz}$ \\
Antenna scan rate & $7.2^{\circ} \mathrm{s}^{-1}$ \\
Pulse duration & $2 \mu \mathrm{s}$ \\
Range-gate spacing & $300 \mathrm{~m}$ \\
\hline
\end{tabular}



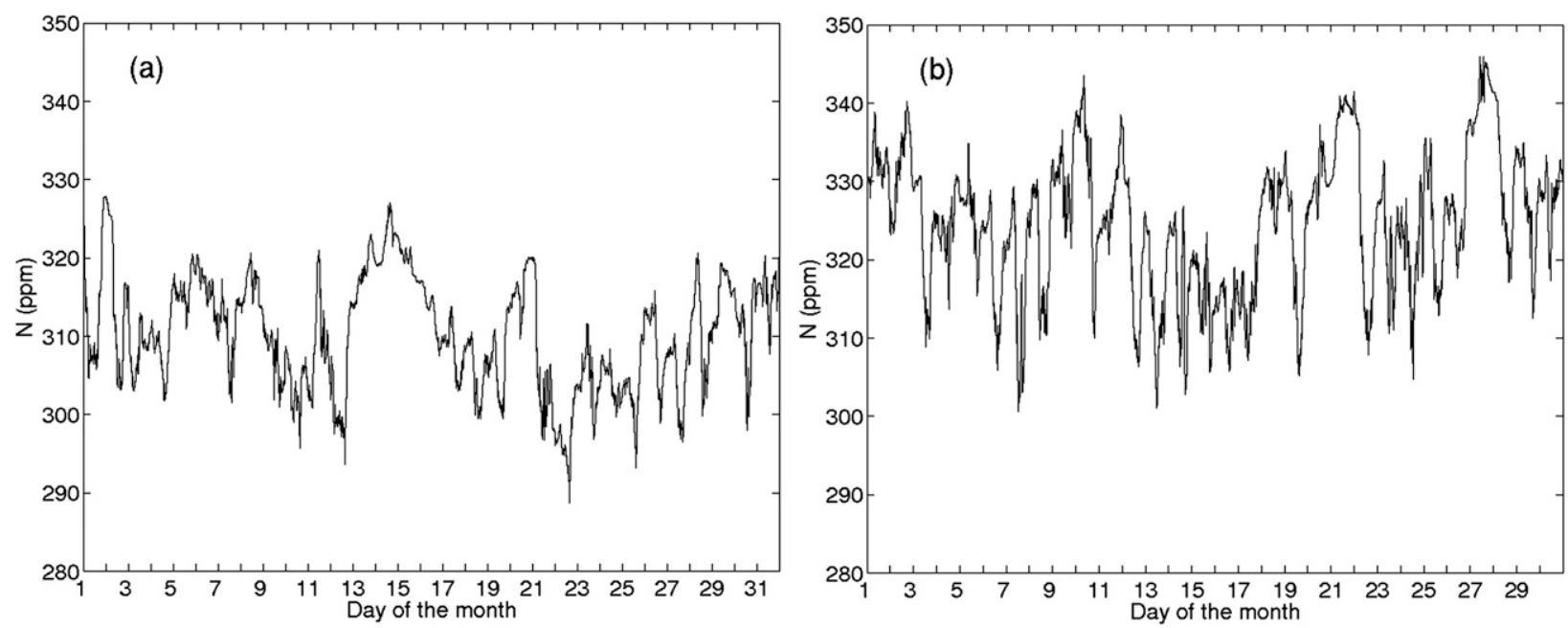

FIG. 3. Refractivity time series from surface observations at Dunkeswell for (a) March 2008 and (b) June 2008.

a phase change correction $\left(\Delta \phi_{\text {gate,corr }}\right)$ may be added at each range gate, as expressed in (8), prior to the standard refractivity retrieval processing (Nicol et al. 2008):

$$
\Delta \phi_{\text {gate, corr }}=\frac{4 \pi r_{\text {gate }} \Delta f_{\mathrm{LO}}}{c} .
$$

To demonstrate the effect of LO frequency changes, we consider phase changes from a target close to the radar at an azimuth of $105^{\circ}$ and a range of $1.35 \mathrm{~km}$. Over $1.35 \mathrm{~km}$, a refractivity change of $1 N$ unit equates to a phase change of about $20^{\circ}$ at $\mathrm{C}$ band, so aliasing would only occur for absolute changes greater than $9 \mathrm{~N}$ units in $5 \mathrm{~min}$; from the nearby Dunkeswell observations in March 2008, this is unlikely.

Two days have been selected: on the first day (2 March 2008), Fig. 4 shows that the refractivity at Dunkeswell
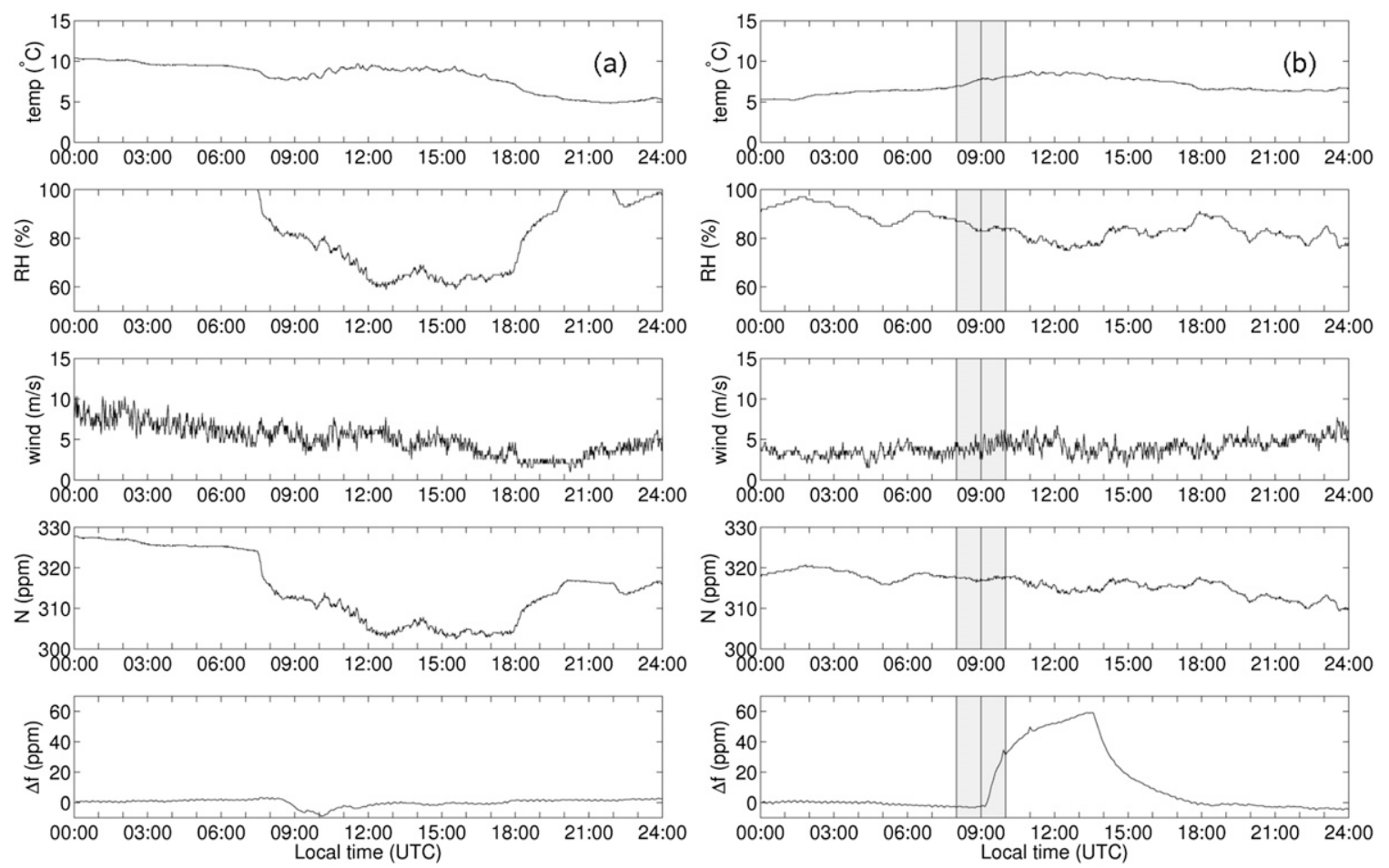

FIG. 4. Time series of temperature, RH, 10-m wind speed, and refractivity $(N)$ at Dunkeswell (20 km, southeast of the radar) and the radar transmitter frequency change (ppm) for (a) 2 Mar 2008 and (b) 6 Mar 2008. The consecutive hour-long periods analyzed in section 5b are shaded in (b). 

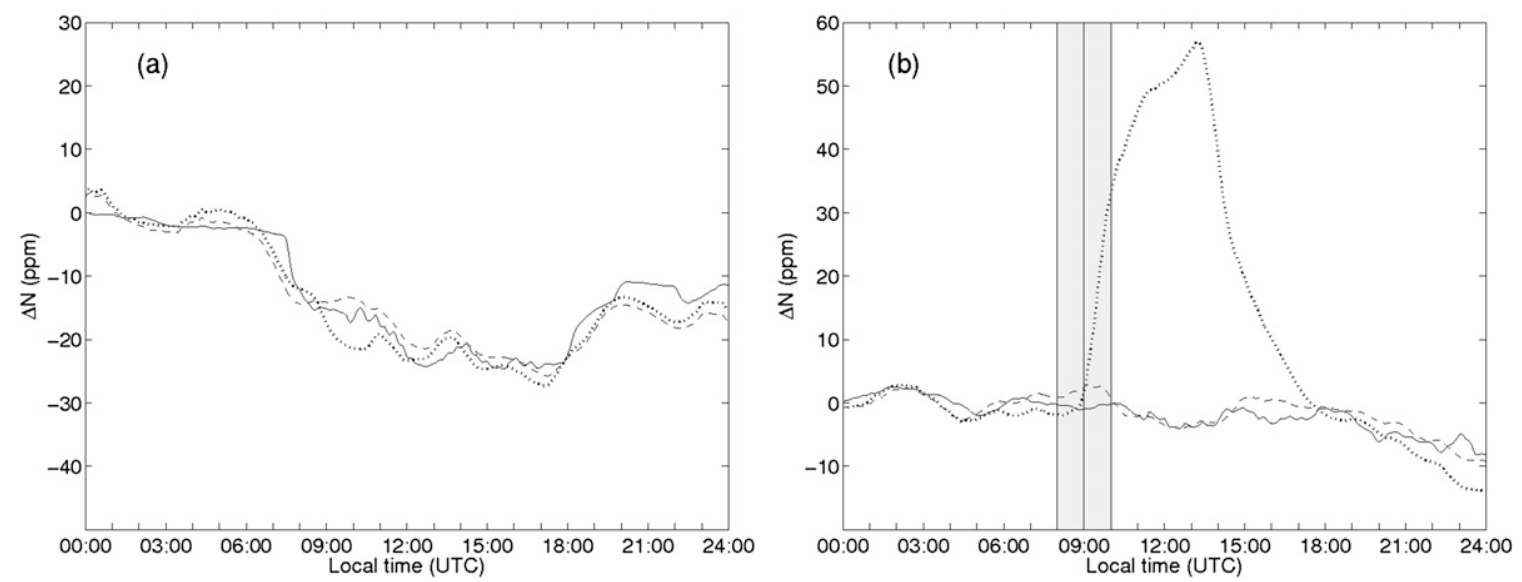

FIG. 5. Time series of refractivity derived with and without correcting for local oscillator changes for (a) 2 Mar 2008 and (b) 6 Mar 2008. Radar refractivity changes have been estimated from a target at $1.35 \mathrm{~km}$ and the accumulated 5-min changes are shown for both the raw phase (dotted line) and the LO frequency-corrected phase (dashed line) using (6). Surface observations (solid line) of refractivity are shown for comparison. The consecutive hour-long periods analyzed in section $5 \mathrm{~b}$ are shaded in (b).

fell by about $20 \mathrm{~N}$ units during the daytime, but the magnetron frequency was fairly constant, whereas on the second day (6 March 2008), the frequency rose by $60 \mathrm{ppm}$ in 4 hours, but the refractivity changed much more slowly. Figure 5 displays the radar refractivity changes derived from the target at a $1.35-\mathrm{km}$ range using the raw phase (dotted line) and the phase corrected for LO frequency changes (dashed line) using (8). For each day the 5-min refractivity changes since midnight have been accumulated and added to the surface observation at midnight to avoid aliasing. Considering that the surface observations are actually made about $20 \mathrm{~km}$ from the radar site, very good agreement with estimates from the LO frequency-corrected phase changes is found in both cases. In the first case, LO frequency changes are moderate and mainly affect measurements for a few hours around 1000 UTC. In the second case, if the raw phases were used, spurious $N$ changes of $60 \mathrm{~N}$ units would be introduced by the very large $(\sim 330 \mathrm{kHz}$; $60 \mathrm{ppm}$ ) LO frequency change, but these are completely removed when the phase corrections in (6) are applied and accurate refractivity changes are retrieved. This figure clearly displays the effect of LO frequency changes as they are continually adjusted throughout the day, serving to validate (4) and (8) in PC2012.

Exactly when and how the LO frequency is changed depends on the implementation of the AFC. Clearly, if the LO frequency were to be held constant, no correction would be required. However, the sensitivity of the radar may be degraded as transmitter frequency drifts could result in the received signal shifting away from the center of the IF filters or the digital filters at baseband. Whenever the LO frequency has changed, corrections must be made to allow useful refractivity retrievals. Any difference between the actual $\left(\Delta f_{\mathrm{LO}}\right)$ and recorded $\left(\Delta f_{\mathrm{LO}}^{\mathrm{rec}}\right)$ LO frequency change $\left(\varepsilon_{\Delta f_{\mathrm{LO}}}=\Delta f_{\mathrm{LO}}-\Delta f_{\mathrm{LO}}^{\mathrm{rec}}\right)$ will result in an error in the estimation of $\Delta N$, given by $\left(\varepsilon_{\Delta f_{\mathrm{LO}}} / \Delta f_{\mathrm{LO}}\right) 10^{6}$. In the next section, we shall evaluate the accuracy of corrections for LO frequency changes.

\section{Quantifying the accuracy of LO frequency changes}

The phase of ground clutter returns may be highly correlated across adjacent range gates; this can occur when clutter targets straddle adjacent gates. The radial extent of returns from highly reflective targets is largely determined by the filtering applied in the radar receiver and may span several range gates (Nicol and Illingworth 2013); we shall refer to these returns as spreading targets. We shall now demonstrate that these returns may be used to estimate the accuracy of corrections for NCO frequency changes through comparison with independent real-time measurements of the transmitter frequency mixed down to the IF.

Laboratory tests have been undertaken to confirm the accuracy of requested STALO frequency changes. A Rohde and Schwartz spectrum analyzer (FSH8) with a Rohde and Schwartz signal generator (SMA100A) as a frequency reference source was used to measure the STALO output frequency as a function of the requested frequency with a $10-\mathrm{Hz}$ bandwidth. The requested frequency was increased and then decreased between 5.59 and $5.62 \mathrm{GHz}$ with frequency steps between $100 \mathrm{kHz}$ and $1 \mathrm{MHz}$. The difference between the requested frequency and the frequency measured by the spectrum analyzer was constant at $8.57 \mathrm{kHz}$ throughout. While this represents an error of $\sim 1.5 \mathrm{ppm}$, the fact that this 
error remained constant within the accuracy of the spectrum analyzer $(10 \mathrm{~Hz})$ as the STALO was stepped through multiple frequencies indicates that requested STALO frequency changes should have negligible effects on radar refractivity retrievals. The use of an ovenized crystal oscillator in the STALO used here should also prevent significant frequency drifts due to changes in the ambient temperature. As refractivity retrievals require very precise LO frequency changes, it is important that the accuracy of each local oscillator stage is verified.

\section{a. Estimating transmitter frequency changes from spreading ground clutter targets}

The return from a spreading target appears in two gates, but the path of the radar wave is identical for both gates. If $f_{\mathrm{Tx}}$ and $f_{\mathrm{LO}}$ are constant, the phase change between two scans at each of the two gates must be equal and the inferred refractivity change from (3) will always be zero. If the frequencies change then from (4), noting that the target location difference is given by $\delta_{\text {gate }+1}-$ $\delta_{\text {gate }}=-\Delta r_{\text {gate }}$ where $\Delta r_{\text {gate }}$ is the range-gate spacing, we have

$\Delta \phi_{\text {gate }+1}-\Delta \phi_{\text {gate }}=-\frac{4 \pi \Delta r_{\text {gate }}}{c}\left(\Delta f_{\mathrm{LO}}-\Delta f_{\mathrm{Tx}}\right)$.

With respect to Fig. 1, a target straddling two gates is depicted by the target toward the edge of the range gate (symbol x) with the return signal $R_{\mathrm{x}}$. The propagation delay is identical for the returns from a single target sampled at a farther range gate, so the returned signal is unchanged though it is now sampled toward the back edge of the pulse rather than toward the front edge. The increase in phase from the front to the back of the transmitted pulse will be similar to the decrease in phase from one range gate to the next from the LO frequency. If there is any difference between the transmitter and LO frequencies, there may be a phase difference at adjacent gates though this will remain constant if both frequencies are unchanged.

The phase change difference from spreading targets will be close to zero for the radar considered here, as the LO frequency is set to match the transmitter frequency immediately prior to each scan through fine adjustments of the NCO, so that $\Delta f_{\mathrm{Tx}} \approx \Delta f_{\mathrm{LO}}$. Because of this, Nicol and Illingworth (2013) established that spreading targets may be recognized by the phase correlation between adjacent range gates; values exceeding 0.95 when averaged over the 288 individual PPI scans for a single dry day (12 December 2007) identified 1053 range-gate pairs associated with spreading targets.

We shall now consider that measured phase changes have been corrected for LO frequency changes using
(8), so it follows from (9) that the transmitter frequency changes estimated from spreading targets $\left(\Delta f_{\mathrm{Tx}}^{\mathrm{spr}}\right)$ is given by

$$
\begin{aligned}
\Delta f_{\mathrm{Tx}}^{\mathrm{spr}}= & \frac{c\left(\Delta \phi_{\text {gate }+1}-\Delta \phi_{\text {gate }}\right)}{4 \pi \Delta r_{\text {gate }}}+\varepsilon_{\Delta f_{\mathrm{STALO}}}+\varepsilon_{\Delta f_{\mathrm{NCO}}} \\
& +\varepsilon_{\Delta f_{\text {Txdritt }}}
\end{aligned}
$$

The first three error terms $\left(\varepsilon_{\Delta f_{\mathrm{STALO}}}, \varepsilon_{\Delta f_{\mathrm{NCO}}}\right.$, and $\left.\varepsilon_{\Delta f_{\mathrm{TXdrift}}}\right)$ relate directly to errors in the nominal STALO frequency, the NCO frequency, and those due to drifts in the transmitter frequency during the scan. Although care has been taken to select only highly correlated pairs of range gates, an additional source of error arises because of the possible inclusion of weak independent returns with the spreading targets. These effects are not simple to quantify as they depend on the relative magnitude and phase of the two (or more) returns; the distribution of errors may be highly skewed. However, such errors are unbiased and tend to zero when averaged over many range-gate pairs.

For the radar considered in this work, with a rangegate separation of $300 \mathrm{~m}$ and phase changes expressed in radians, transmitter frequency changes have been estimated from

$$
\Delta f_{\mathrm{Tx}}^{\mathrm{spr}}=79.5 \overline{\left(\Delta \phi_{\text {gate }+1}-\Delta \phi_{\text {gate }}\right)} \mathrm{kHz} .
$$

Here, the mean phase change difference is calculated over all (1053) adjacent range-gate pairs associated with spreading targets. As a phase change difference of $1^{\circ}$ results from a frequency change of approximately $1.4 \mathrm{kHz}$, aliasing occurs when absolute frequency changes exceed about $250 \mathrm{kHz}$. Hourly frequency changes have been calculated using (11) for March 2008 (derived from scans every $5 \mathrm{~min}$ ). Such estimates are unaffected by target motion such as wind-induced swaying as phase changes are equally affected since we are considering the same target at each range gate, though no attempt has been made to avoid periods of precipitation or radar artifacts such as interference.

In Fig. 6a, these hourly frequency changes $(>8000)$ are compared with those obtained from independent estimates of the transmitter frequency made in real time at IF (as described in section 3). A histogram of the differences between the two estimates of the frequency change is displayed in Fig. 6b. The agreement is remarkably good, which supports the model of spreading targets; the returns from dominant point targets replicate the transmitted pulse. The rms discrepancy between the hourly frequency change estimates throughout the 

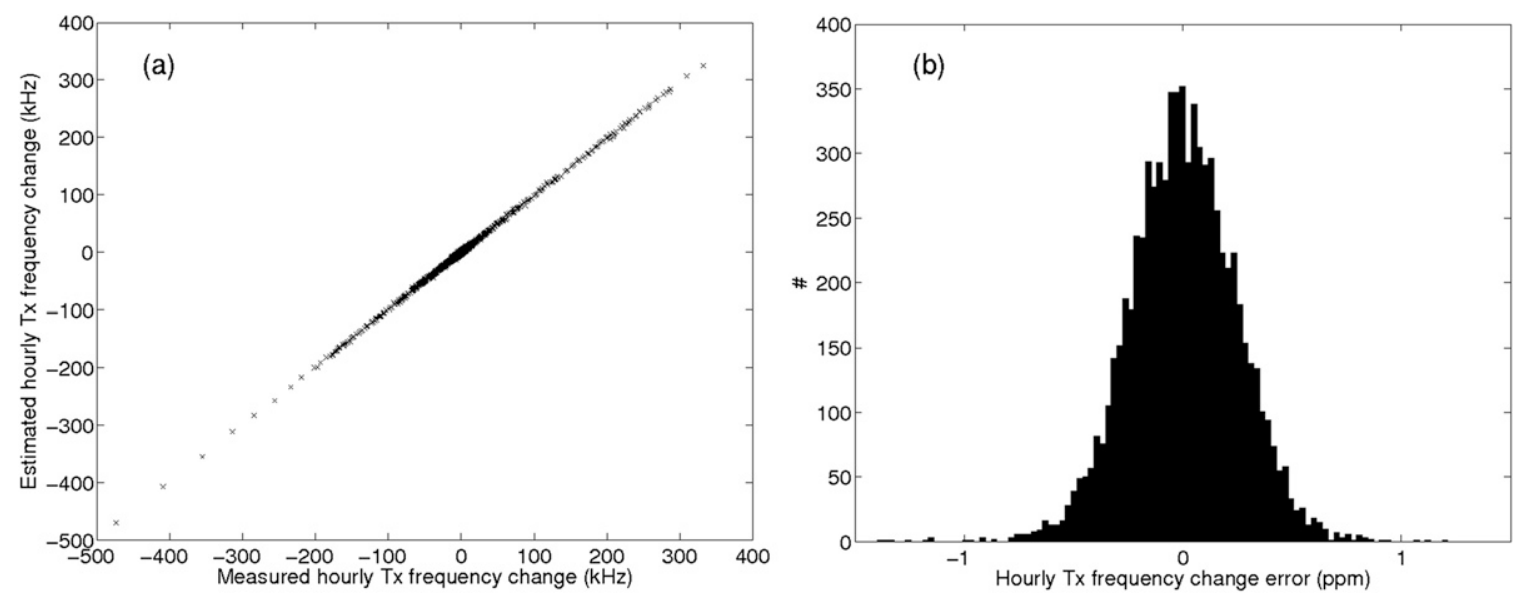

FIG. 6. (a) Comparison of hourly changes (over 8000 in total) in the magnetron transmitter frequency measured in real time and estimates derived from spreading targets (1053 range-gate pairs) using (11) during March 2008 and (b) the histogram of the discrepancy between these two measures in parts per million. The rms discrepancy $(0.25 \mathrm{ppm})$ indicates that $\Delta f_{\mathrm{NCO}}$ can be requested and is known very precisely.

month is $0.25 \mathrm{ppm}$ with daily values ranging from 0.21 to $0.29 \mathrm{ppm}$; these estimates are essentially unbiased (mean discrepancy $=0.002 \mathrm{ppm}$ ).

Any error in the STALO frequency change will equally affect the real-time measurements of $f_{\mathrm{Tx}}$ made at IF and those made from spreading targets. The discrepancy between these two estimates will then have contributions from the final three sources of error expressed in (10), along with any errors in the real-time transmitter frequency measurements or due to the possible inclusion of weak independent returns. Assuming these sources of error are independent, each of these errors must be less than the observed rms discrepancy of just $0.25 \mathrm{ppm}$ (most importantly $\varepsilon_{\Delta f_{\mathrm{NCO}}}$ due to the direct effect on refractivity errors). Therefore, the rms error in refractivity changes due to the continuously adjusted NCO frequency from scan by scan must be no larger than $0.25 \mathrm{~N}$ units. This confirms that the fine-tuned frequency adjustments of the $\mathrm{NCO}$ will not adversely affect refractivity retrievals. In addition, the real-time transmitter frequency measurements at IF are very accurate, and the methodology of selecting spreading targets is sufficiently robust.

The comparisons presented here relate to frequency measurements at IF (real-time measurements) and at baseband (spreading targets). In the absence of direct real-time $f_{\text {Tx }}$ measurements at radio frequency, it is impossible to operationally verify the accuracy of STALO frequency changes. However, laboratory measurements indicate that requested STALO frequency changes are more than sufficiently accurate $(<0.002 \mathrm{ppm})$. In addition, hourly refractivity changes derived from this radar were found to have rms differences of just $1.25 \mathrm{~N}$ units in comparison with in situ measurements over a 5-month period (Nicol et al. 2013). These differences are expected to be dominated by spatial and temporal representativeness and the stability of the STALO frequency is presumably very good.

A similar approach was used to validate the expression for phase changes in PC2012 using a single set of adjacent range gates. However, an important distinction is that the real-time frequency measurements in PC2012 were made at baseband rather than at IF (as they are here). As such, errors in not only the STALO frequency but also in the down conversion from IF to baseband would not be revealed in their comparisons. The relatively poor agreement (33\% error) between the observations $\left(0.24^{\circ} \mathrm{kHz}^{-1}\right)$ and theory $\left(0.36^{\circ} \mathrm{kHz}^{-1}\right)$ in PC2012 is presumably because the returns across successive range gates were not perfectly correlated and therefore not absolutely from the same target (PC2012, p. 1433).

\section{b. Implications of spreading target for refractivity retrievals}

Refractivity changes from spreading targets may then be expressed in terms of the fractional transmitter frequency change (in ppm) $\Delta F_{\mathrm{Tx}}=\left(\Delta f_{\mathrm{Tx}} / f_{\mathrm{Tx}}\right) 10^{6}$ by substituting (9) into (3) as

$$
\Delta N=-\Delta F_{\mathrm{Tx}} .
$$

For radars with klystron transmitters, spreading targets will lead to identical phase changes at adjacent gates. Their inclusion tends to bias estimated refractivity changes toward zero; when the standard approach using pulse-pair processing of phase changes in (3) prior to any smoothing is used to estimate the field-averaged 
refractivity change (Nicol and Illingworth 2013). For radars with magnetron transmitters, these biases tend toward the fractional change in the transmitter frequency once the measured phase changes have been corrected for LO frequency changes.

\section{Phase change noise due to target location uncertainty}

In this section, we first derive a theoretical expression for the random phase noise arising from the uncertainty of the target location relative to the range-gate center when transmitter frequency changes occur, the Tx target location noise. Observations made at $\mathrm{C}$ band are then used to quantify the target location uncertainty and the magnitude of this noise. These results allow us to predict the phase change noise due to target location uncertainty, the refractivity target location noise-when refractivity changes occur affecting both magnetron and klystron systems. For large $\Delta N$, this random phase noise can prevent accurate retrievals and, even at $\mathrm{S}$ band, may limit the use of a reference field with refractivity values very different from those being observed.

\section{a. Theoretical phase change noise introduced by transmitter frequency changes}

The effect of transmitter frequency changes may be isolated by setting $\Delta n=0$ and $\Delta f_{\mathrm{LO}}=0$, so (4) becomes

$$
\varepsilon_{\Delta \phi_{\text {gate }}}=\Delta \phi_{\text {gate }}=-\frac{4 \pi \delta_{\text {gate }} \Delta f_{\text {Tx }}}{c} .
$$

The phase change error term in (13) increases with $\Delta f_{\mathrm{Tx}}$ and $\delta_{\text {gate, }}$, so unlike (8) this effect does not progress linearly with range but varies from gate to gate depending on the precise target location relative to the range-gate center. In contrast to LO frequency changes, transmitter frequency changes do not directly bias estimated refractivity changes, but they introduce a significant source of phase change noise for magnetron-based radars. The rms target location phase change noise may be expressed in terms of the standard deviation of the target location uncertainty $\left(\sigma_{\delta_{\text {gate }}}\right)$ as

$$
\sigma_{\Delta \phi}=\frac{4 \pi \sigma_{\delta_{\text {gate }}\left|\Delta f_{\mathrm{Tx}}\right|} .}{c} .
$$

This effect is independent of the nominal operating radar frequency or band (e.g., X, C, or S band). However, if the fractional change in frequency is proportional to the change in temperature for magnetron transmitters (due to thermal expansion), then these effects should be more severe at higher frequencies (shorter wavelengths).
Measurements of the transmitter frequency at $\mathrm{C}$ band presented in section 3a indicate that although a $\Delta f_{\mathrm{Tx}}$ as large as $200 \mathrm{kHz}$ are relatively infrequent over an hour, changes of up to $300 \mathrm{kHz}$ (Fig. 2b) occurred during the day throughout the summer. If $\sigma_{\delta_{\text {gate }}}=75 \mathrm{~m}$ then (14) predicts an additional phase change noise (in degrees) equal to $\Delta F_{\mathrm{Tx}}$ (in ppm).

\section{b. Observed phase change noise from transmitter frequency changes}

To quantify the additional phase change noise when $f_{\text {Tx }}$ changes, we will analyze observed phase change noise for two consecutive hours when the refractivity changes are very small and $f_{\mathrm{Tx}}$ is constant during the first hour but has large changes in the second hour. The refractivity and frequency changes for such a 2-h period on 6 March 2008 are shaded in Fig. 4b. The refractivity was relatively constant throughout both periods, and because the weather was overcast we can assume that this was true within a distance of $30 \mathrm{~km}$ from the radar; the transmitter frequency was relatively constant during the first hour (0800-0900 UTC) but increased by $34 \mathrm{ppm}$ ( $\sim 190 \mathrm{kHz})$ during the second hour (0900-1000 UTC). Phase change measurements relative to the beginning of each period were calculated every $10 \mathrm{~min}$ out to $30 \mathrm{~km}$ using only range gates $\left(1^{\circ} \times 300 \mathrm{~m}\right)$ with significant returns $(\mathrm{dBZ}>15)$; only range gates where the median power ratio (Nicol and Illingworth 2013) was greater than 0.8 throughout the day were accepted as being stationary. Phase changes were then corrected for LO frequency changes using (8).

Phase change noise has been estimated from the local standard deviation of the LO-corrected phase changes $\left(\sigma_{\Delta \phi}\right)$ over areas of $3.9 \mathrm{~km}$ in range by $13^{\circ}$ in azimuth around each pixel with the approach followed in Park and Fabry (2010), originally applied to angular data (wind direction) by Weber (1997):

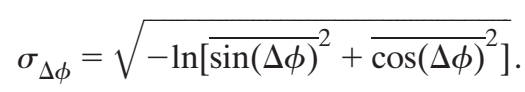

Unlike linear variables, difficulties arise in estimating angular standard deviations due to variable integration limits and aliasing; however, (15) can provide robust estimates of the angular standard deviation based on the temporal persistence or, here, the spatial consistency of the data. The size of the area $\left(3.9 \mathrm{~km} \times 13^{\circ}\right)$ was selected to be large enough to provide proper statistics in (15) though not so large as to be unduly influenced by refractivity changes. This approach requires that refractivity changes are very small, as an additional noise of about $15^{\circ}$ would be expected over a range of $3.9 \mathrm{~km}$ for $\Delta N=1$. 
To estimate refractivity changes throughout these periods, the LO-corrected phase changes are first smoothed using triangular functions with a $1.5-\mathrm{km}$ base in range and a $4-\mathrm{km}$ base in azimuth. Particularly at shorter wavelengths, the radial extent of the smoothing kernel must be limited or refractivity changes are likely to be underestimated (Nicol and Illingworth 2013). Pulse-pair estimates of the gate-to-gate phase change differences averaged over areas of $3.9 \mathrm{~km}$ in range by $13^{\circ}$ in azimuth around each pixel were finally used to estimate refractivity changes from (3).

Figures $7 \mathrm{a}$ and $7 \mathrm{~b}$ show the phase change noise and refractivity changes, respectively, at $0810,0820,0830$, 0840, 0850, and 0900 UTC relative to 0800 UTC. Refractivity changes tend to become noisy and unreliable when the phase change noise exceeds about $95^{\circ}$ for the spatial smoothing of phase changes applied here; these regions have been removed from the corresponding refractivity plots in Fig. 7c. It may be noted that apart from regions with high phase change noise, reliable refractivity estimates have been obtained over the majority of the ground clutter field. In addition, there is only a slight degradation as the time separation increases from 10 to $60 \mathrm{~min}$. The rms phase change noise over the entire field (shown above each phase noise image) increased from $86^{\circ}$ at $0810 \mathrm{UTC}$ to $90^{\circ}$ at $0900 \mathrm{UTC}$. Since the transmitter frequency changes by less than $1 \mathrm{ppm}$, it is likely that this slight increase in phase change noise is due to small refractivity changes (e.g., $|\Delta N| \approx 2$ ).

The corresponding images at 0910, 0920, 0930, 0940, 0950, and 1000 UTC relative to 0900 UTC are shown in Figs. $8 \mathrm{a}, 8 \mathrm{~b}$, and $8 \mathrm{c}$. In contrast to Fig. $7 \mathrm{a}$, a progressive increase in phase change noise is observed throughout the hour, although there was no significant change in wind speed or refractivity during this period that could explain this increase (see Figs. $4 \mathrm{~b}, 5 \mathrm{~b}$ ). The rms phase change noise over the entire field was $88^{\circ}$ at 0910 UTC $\left(\Delta F_{\mathrm{Tx}}=0 \mathrm{ppm}\right)$ and progressively increased to $110^{\circ}$ at $1000 \mathrm{UTC}\left(\Delta F_{\mathrm{Tx}}=34 \mathrm{ppm}\right)$. Subtracting the rms phase change noise at 0910 UTC (in terms of variance) from the later values allows the component of phase change noise explained by $\Delta F_{\mathrm{Tx}}\left(\sigma_{\Delta \phi \mathrm{f}}\right)$ to be estimated. Based on this, $\sigma_{\Delta \phi \mathrm{f}}$ increased progressively from $0^{\circ}$ to $66^{\circ}$ between 0910 and $1000 \mathrm{UTC}$ as $\Delta F_{\mathrm{Tx}}$ increased from 0 to $34 \mathrm{ppm}$; the correlation between $\sigma_{\Delta \phi \mathrm{f}}$ and $\Delta F_{\mathrm{Tx}}$ during this period was 0.96 . By rearranging (14), the extra $66^{\circ}$ of phase change noise due to target location uncertainty and a transmitter frequency change of $190 \mathrm{kHz}$ (34 ppm) implies that the rms target location uncertainty was about $150 \mathrm{~m}$. As target location uncertainty is presumably proportional to range resolution $(L)$, which is determined by the pulse duration $\tau(L=c \tau / 2)$, we may note that the estimated rms target location uncertainty determined here is approximately equal to $L / 2$.

While this suggests that the majority of clutter targets are located within the range gate at which they are sampled, a proportion of ground clutter returns correspond to targets in adjacent range gates. This is consistent with spreading targets (considered in section 4) and supported by observations of high phase correlations over distances up to four range gates in Nicol and Illingworth (2013). This implies that target location uncertainties can at times be as large as two range gates $(600 \mathrm{~m}$ for the radar considered here), though typically no larger.

The rapid transmitter frequency change observed here corresponded to a radar site visit by engineers. The frequency change seems too large and abrupt to be caused solely by temperature effects and is likely due to changes in power usage at the site. Although changes such as this are not typical, decreases of similar magnitude are often observed during the day in summer as the temperature of the radar cabin increases (Fig. 2b).

\section{c. Theoretical phase change noise introduced by refractivity changes}

When a target is not at the range-gate center, $\delta_{\text {gate }}$ is not zero in the refractivity term of (4), which results in a phase change error $\left(\varepsilon_{\Delta \phi_{i}}\right)$ in proportion to changes in refractive index:

$$
\varepsilon_{\Delta \phi_{i}}=-\frac{4 \pi f_{\mathrm{Tx}} \delta_{\text {gate }} \Delta n}{c} .
$$

The analysis of phase change noise due to transmitter frequency changes in the previous section indicated that the rms target location uncertainty was about half the range resolution or range-gate length $\left(\sigma_{\delta} \approx L / 2\right)$. The rms phase change noise due to refractivity changes is then given by

$$
\sigma_{\Delta \phi} \approx \frac{2 \pi L f_{\mathrm{TX}}|\Delta n|}{c} .
$$

In contrast to the phase change noise due to transmitter frequency changes in (14), this noise is proportional to the operating frequency in addition to the pulse duration. For a pulse duration of $2 \mu \mathrm{s}(L=300 \mathrm{~m})$ and refractivity change of $10 \mathrm{~N}$ units, the additional phase change noise would be approximately $36^{\circ}, 20^{\circ}$, and $11^{\circ}$ at $\mathrm{X}, \mathrm{C}$, and $\mathrm{S}$ bands, respectively. In warmer climates, particularly when using a reference period, the large refractivity changes that may develop can result in significant phase change noise even at $\mathrm{S}$ band; for the NEXRAD radars $(L=250 \mathrm{~m})$, refractivity changes of $60 \mathrm{~N}$ units would result in about $55^{\circ}$ additional phase change noise. Combined with phase noise due to target motion, for example, this can result in a degradation of refractivity retrievals. 
a
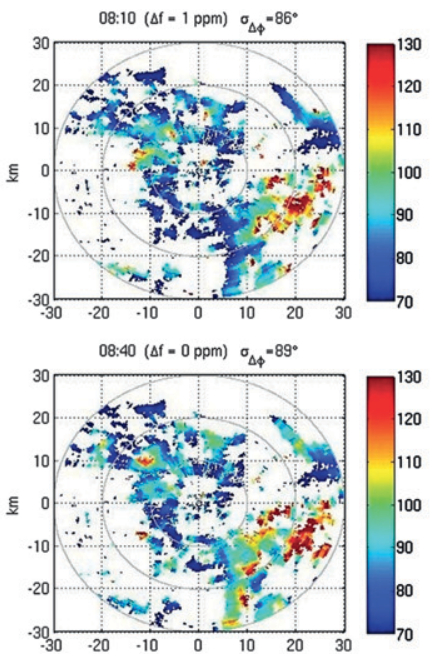

b
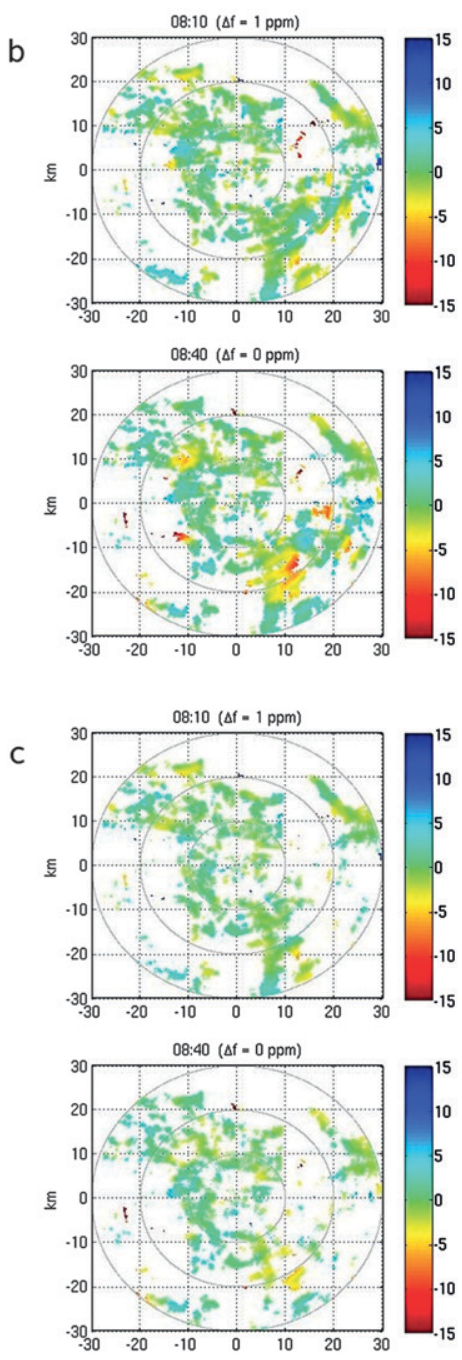
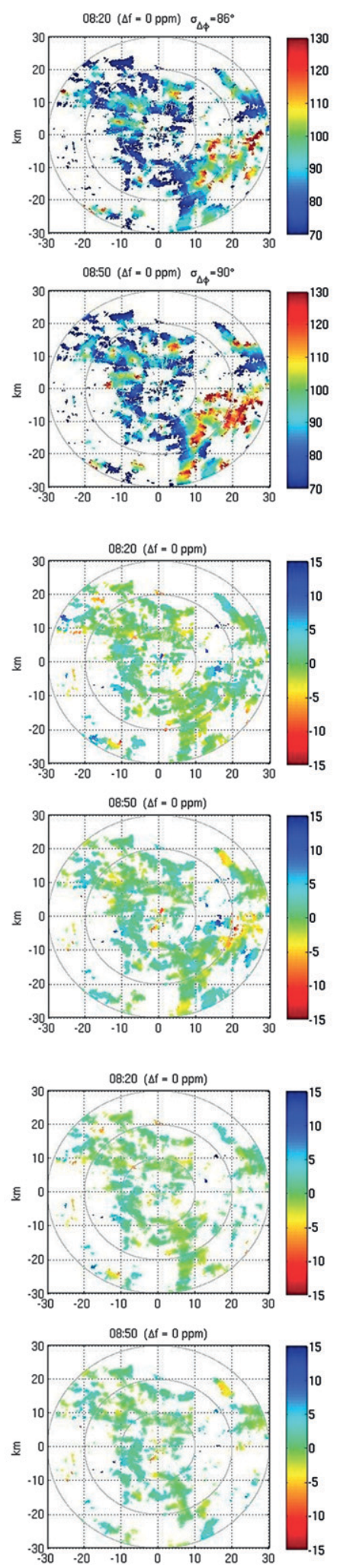
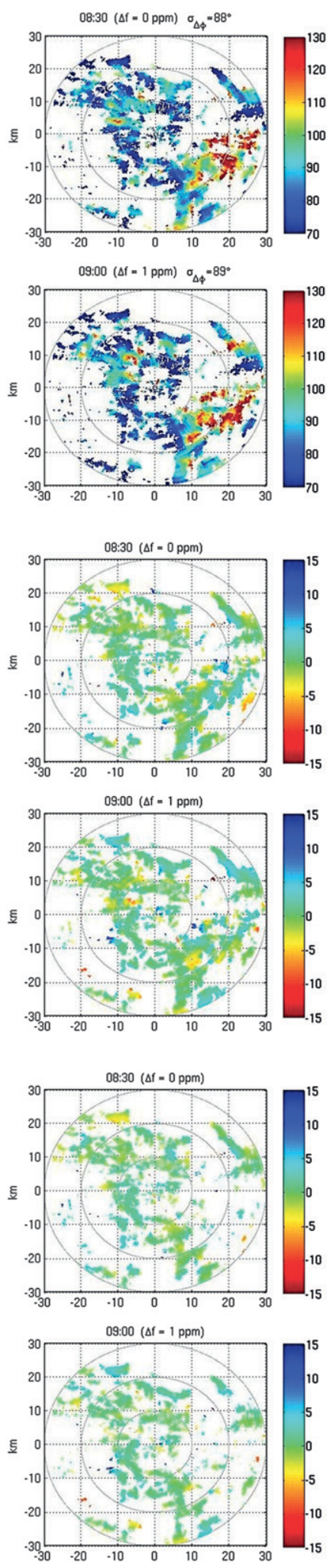

FIG. 7. Phase change noise in (a) degrees, (b) refractivity changes, and (c) refractivity changes excluding unreliable retrievals (phase change noise $>95^{\circ}$ ) during a period with little change in refractivity or magnetron transmitter frequency $\left(\Delta f_{\mathrm{Tx}}\right)$. Observations are averaged over areas of $3.9 \mathrm{~km}$ in range and $13^{\circ}$ in azimuth from 0810-0900 UTC relative to 0800 UTC (6 Mar 2008). The rms phase change noise $\left(\sigma_{\Delta \phi}\right)$ remains relatively constant throughout the hour. 
a
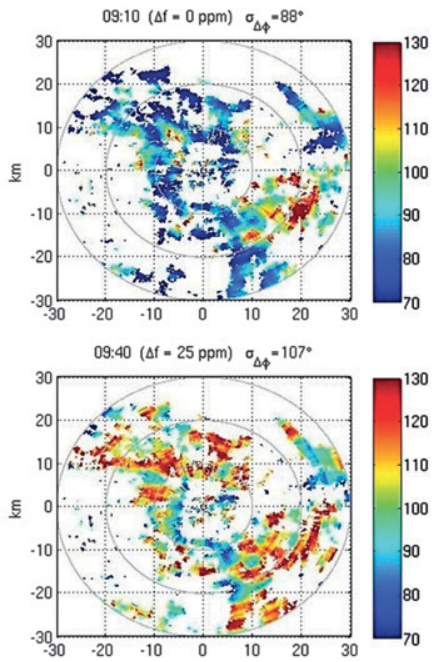

b
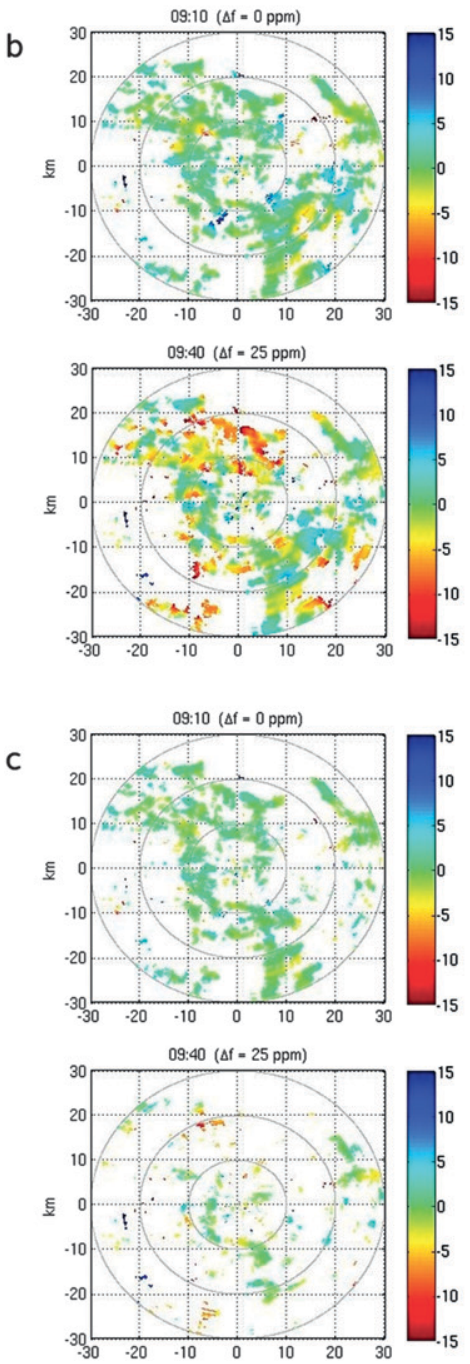
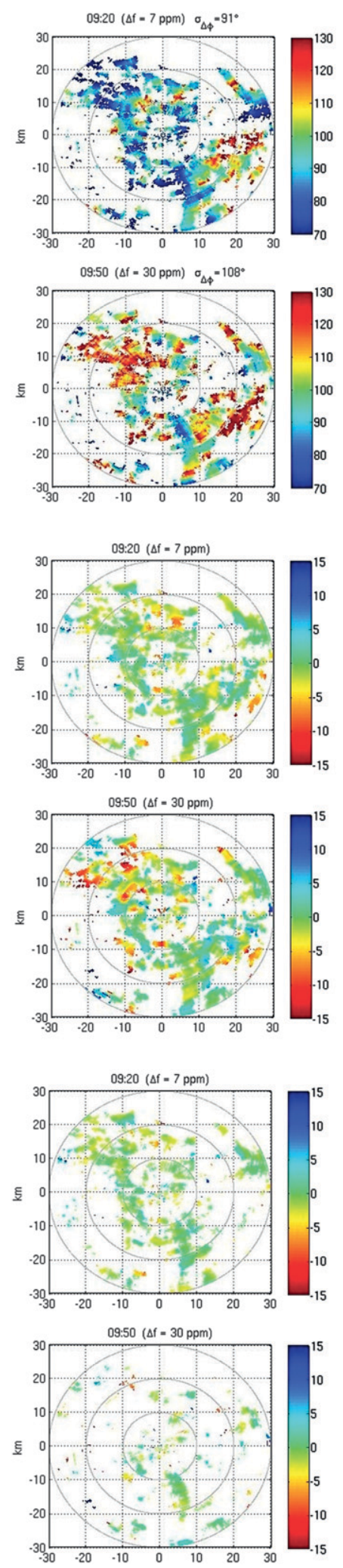
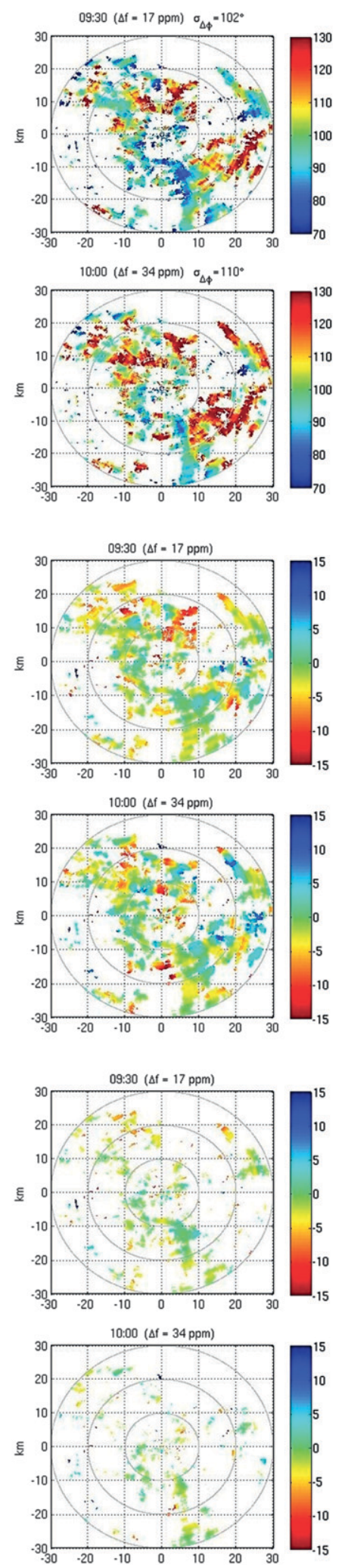

FIG. 8. As in Fig. 7, but for changes from 0910-1000 UTC relative to 0900 UTC (6 Mar 2008). Refractivity changes were again small, though large transmitter frequency changes $\left(\Delta f_{\mathrm{Tx}}\right)$ occurred. The rms phase change noise $\left(\sigma_{\Delta \phi}\right)$ increases progressively with $\Delta f_{\mathrm{Tx}}$ throughout the hour (correlation $=0.96$ ). 


\section{Reducing target location uncertainty}

In the previous section, we have demonstrated that target location uncertainty may result in significant phase change noise due to both refractivity and transmitter frequency changes. We shall now consider how the effects of target location uncertainty may be reduced.

\section{a. Consideration of pulse length}

Figure 8 demonstrates that transmitter frequency changes of $34 \mathrm{ppm}$ lead to a disastrous loss of refractivity data because of phase noise arising from the uncertainty of the target location within the gate. Transmitter frequency changes at the $\mathrm{C}$ band of $400 \mathrm{kHz}(70 \mathrm{ppm})$ can develop in time (Fig. 2) and should introduce phase change noise close to $140^{\circ}$ with a pulse duration of $2 \mu \mathrm{s}$. Refractivity changes based on surface observations near the radar at Cobbacombe may be as large as $40 \mathrm{~N}$ units based on Fig. 3, implying additional noise approaching $80^{\circ}$. An increase in temperature tends to decrease both the transmitter frequency and refractivity values, so these effects are not independent and the total phase change noise could exceed $200^{\circ}$. Even in the absence of other sources of phase change noise (e.g., target motion), this noise will prevent retrievals using a reference field.

However, if we now consider the retrieval of 1-h refractivity changes, the maximum phase change noise may be reduced down to about $55^{\circ}$, comprising $35^{\circ}$ $(<100 \mathrm{kHz})$ and $20^{\circ}(<10 \mathrm{ppm})$ from frequency and refractivity changes, respectively. Although phase change noise also arises from target motion and changes in the vertical gradient of refractivity $(d n / d h)$ (Park and Fabry 2010), 1-h refractivity changes obtained using the operational C-band magnetron weather radar considered in this work have shown good agreement with surface observations, even during the summer months (Nicol et al. 2012). Naturally, phase change noise may be reduced further by deriving refractivity changes between adjacent radar scans (e.g., over $5 \mathrm{~min}$ ). However, to derive changes over longer periods, these changes must be integrated and the associated estimation errors must progressively increase. The tradeoff implied here is the subject of future studies.

The benefits of using shorter pulses for refractivity retrieval have been considered in Nicol and Illingworth (2013) for radars with klystron transmitters, including the reduction in refractivity target location noise. For magnetron transmitter radars, this noise reduction is even more important due to the additional Tx target location noise. Relative to the $2-\mu$ s pulse (300-m range resolution) currently used for refractivity retrievals on the U.K. radar network, a $0.5-\mu$ s pulse ( $75-\mathrm{m}$ range resolution) would directly reduce the magnitude of the phase change noise due to target location uncertainty by $75 \%$. The maximum contribution to phase change noise from target location uncertainty would be reduced to about $50^{\circ}$ and the use of a reference field may then be achievable as such phase change noise seems tolerable when deriving 1-h changes. However, the significance of phase change noise due to changes in $d n / d h$ has yet to be determined when using a reference field at shorter wavelengths.

\section{b. Dual-frequency phase measurements for accurate target ranging}

Target location uncertainty could be reduced by making phase measurements at two close but distinct transmitter frequencies (ideally alternating frequencies from pulse to pulse). This would allow targets away from the range-gate center to be identified and discarded. This concept is very similar to frequency domain interferometry (FDI), which was first proposed by Kudeki and Stitt (1987) for very high frequency (VHF) radars to accurately determine the heights and thicknesses of thin persistent layers of refractive index irregularities. As measurements at the two frequencies are effectively simultaneous, $\Delta N=0$ and, assuming that the LO frequency is unchanged, the target location relative to the range-gate center may be derived from (7) as

$$
\delta_{\text {gate }}=-\frac{c}{4 \pi \Delta f_{\mathrm{Tx}}} \Delta \phi_{\text {gate }} .
$$

By applying pulse-pair calculations between pulses at these two frequencies, the argument of the vector sum provides the phase change, while the magnitude reflects the consistency throughout the measurement period and could be used to discard unreliable estimates. The maximum unambiguous distance from the range-gate center depends only on the frequency step $\Delta f_{\mathrm{Tx}}$ and is independent of the pulse length and the operating frequency:

$$
\delta_{\max }= \pm \frac{c}{4 \Delta f_{\mathrm{Tx}}} .
$$

A maximum unambiguous target distance of about $900 \mathrm{~m}$ would require a frequency shift of $80 \mathrm{kHz}(\approx 14 \mathrm{ppm}$ at $\mathrm{C}$ band), which would allow even the most intense clutter targets to be unambiguously ranged for the operational network radars. Larger frequency changes would be required for more accurate ranging with shorter pulses. This technique could allow targets located far from the center of the range gate to be excluded (reducing phase change noise from large refractivity changes) and spreading targets to be identified in real time (removing the tendency to bias refractivity changes toward zero for 
frequency-stable radars). For distributed targets such as precipitation, the power-weighted target range should be close to the range-gate center. In this case, the alternating frequency should lead to negligible phase changes and would not affect Doppler velocity estimates.

Although precise control of the transmitter frequency is desirable for this technique, an alternative is possible for magnetron transmitters. Successive blocks of pulses at different PRFs result in frequency shifts of about the right magnitude, and a dual-PRF operation such as this is already used in some systems to increase the maximum unambiguous velocity. Alternating blocks of pulses with PRFs of 1.6 and $2.4 \mathrm{kHz}$ produced a shift of about $200 \mathrm{kHz}$ for the $\mathrm{X}$-band magnetrons of the CASA radars (Fig. 9b in Junyent et al. 2010) with a significant change $(\sim 100 \mathrm{kHz})$ occurring in the time of a few pulses. Relatively slow scan rates may then be required to help ensure that the successive bursts of pulses largely encounter the same targets.

\section{Conclusions}

This work has considered two distinct effects of the frequency changes experienced by magnetron transmitters on radar refractivity retrieval. First, LO frequency changes produce a phase change, which progresses regularly with range, identical to that due to spatially uniform refractivity changes. Second, transmitter frequency changes produce phase change noise, which depends on the precise range of the dominant ground clutter target at each range gate. Refractivity estimates will be in error by the fractional LO frequency change (in ppm) if no correction is applied. Even rather small LO frequency changes, if uncorrected, can significantly bias refractivity retrievals, while relatively large transmitter frequency changes are typically required to introduce significant phase change noise. An LO frequency change of $200 \mathrm{kHz}$ would lead to refractivity change errors of about 20, 35, and $65 \mathrm{~N}$ units at $\mathrm{X}$-, $\mathrm{C}$-, and S-band wavelengths, respectively, regardless of the pulse length. However, if the LO frequency is recorded in real time this effect may be accurately corrected for. Laboratory measurements show that STALO frequency changes can be requested with exceptional accuracy $(<0.002 \mathrm{ppm})$.

The phase change gradient across spreading targets (single targets which dominate returns over several range gates) is proportional to any differences in the transmitter and LO frequency changes. Excellent agreement has been found between independent realtime measurements of the transmitter frequency at the IF and estimates from spreading targets. This not only confirms the accuracy of transmitter frequency measurements and baseband down conversion but also indicates that the residual refractivity change errors from the necessary correction for $\mathrm{LO}$ frequency changes is less than $0.25 \mathrm{~N}$ units.

The influence of phase-correlated returns or spreading targets on refractivity retrievals using klystron-based radars was identified by Nicol and Illingworth (2013), who demonstrated a tendency for refractivity changes to be biased toward zero. It is even more important that these targets are excluded when using magnetron-based radars for two reasons. First, the biases tend toward the fractional transmitter frequency change (ppm) rather than zero and more significant refractivity retrieval errors are likely to result. Second, greater phase change errors may be introduced. The exclusion of spreading targets may be achieved by eliminating or penalizing the weaker returns where large reflectivity gradients (e.g., dBZ $>15)$ and high phase correlations (e.g., >0.3) exist between adjacent range gates in ground clutter.

Although the direct effects of transmitter frequency changes are proportional to the pulse length, they are generally less significant than those due to LO frequency changes. A transmitter frequency change of $200 \mathrm{kHz}$ leads to additional phase change noise of about $18^{\circ}, 36^{\circ}$, and $72^{\circ}$ for pulse durations of $0.5,1$, and $2 \mu \mathrm{s}$, respectively, independent of the radar wavelength. However, as LO frequency changes can be accurately corrected for, the primary confounding effect (phase change noise) of transmitter frequency changes relates to target location uncertainty, which may only be reduced by using shorter pulses if the precise target locations are unknown.

The effects described in this paper may also affect radars with klystron transmitters if frequency changes occur because of the replacement of certain components or even when powered off then on again. More generally, target location uncertainty may introduce significant phase change noise from refractivity changes alone when long pulse lengths are used. For example, an additional phase change noise of about $55^{\circ}$ is anticipated for the $250-\mathrm{m}$ gate length of the NEXRAD radars when refractivity changes of $60 \mathrm{~N}$ units have occurred. These effects may be mitigated by alternating between two closely spaced frequencies, which allows the precise range of targets to be determined by the induced phase change. Targets located away from the range-gate center could then be discarded, removing the largest phase change errors. This technique could also be possible with magnetron transmitters operating with a dual-PRF to intentionally modulate the transmitted frequency.

Since the seminal paper on radar refractivity retrieval (Fabry et al. 1997), it has been a common misconception 
that refractivity biases occur directly from transmitter frequency changes rather than LO frequency changes, which is at odds with PC2012 and the findings of this work. Although Bodine et al. (2011) present a correction for transmitter frequency changes, the magnitude of the correction expressed in their Eq. (7) seems to be in error, and differs from that suggested in Fabry et al. (1997) by a factor of $2 \pi$. As a result, the correction applied by Bodine et al. (2011) is significantly underestimated, as the corrections of up to about $3 N$ units in their Fig. 3 should actually be as large as $20 \mathrm{~N}$ units. Better agreement would then have been found if no correction were applied, which implies that the LO frequency was unchanged throughout this $24-\mathrm{h}$ period. The NCO down conversion of the CASA radars they considered is only altered when the estimated frequency has drifted by $250 \mathrm{kHz}$ (Junyent et al. 2009). Such an LO frequency change at $\mathrm{X}$ band would be expected to produce a sudden jump of about $27 \mathrm{~N}$ units in refractivity retrievals, so it seems likely that it was indeed unchanged during this time.

Total phase change noise may have contributions from many sources such as target motion, $d n / d h$ (combined with target height variability), and transmitter frequency and refractivity changes (combined with target location uncertainty). Assuming that the fractional transmitter frequency change is proportional to the change in temperature, all these factors are proportional to the operating frequency and have greater effects at shorter wavelengths. Although the sensitivity to refractivity changes is also proportional to the radar wavelength, problems occur when phase change noise becomes too large. At shorter wavelengths, it is more important both to use a short pulse to reduce errors related to target location uncertainty and to effectively discriminate suitably stationary targets. While Bodine et al. (2011) suggest that "the notion of using a reference time from prior days does not apply when deriving refractivity from a magnetron-based radar," this may indeed be possible if sufficiently short pulses were used. However, the use of a reference period is increasingly difficult at shorter wavelengths and with longer pulses, particularly when using magnetron transmitters.

Acknowledgments. This work has been supported by the NCAS Facility for Ground-Based Atmospheric Measurements (FGAM). The authors thank Mike Edwards at the Met Office for the radar hardware developments and Mike Molyneux for providing synoptic station observations. Thanks to Jon Eastment at the STFC Rutherford Appleton Lab for useful discussions on the radar hardware. This work has been partially funded through the NERC FREE project, Grant NE/ E002137/1.

\section{APPENDIX}

\section{The Phase Change for a Stationary Point Target with Changing Frequency}

The phase change between two pulses at different times for a point target is now derived, taking transmitter and both local oscillator frequency changes into account. For refractivity retrieval, these two times could correspond to PPI scans separated by hours or even months. The radar transmits a pulse with a given frequency $\left(f_{\mathrm{Tx}}\right)$, phase $\left(\phi_{o}\right)$, and amplitude $(A)$ :

$$
T(t)=A(t) e^{j\left(2 \pi f_{\mathrm{Tx}} t+\phi_{o}\right)} .
$$

The amplitude is somewhat arbitrary, though it will ideally be constant over the pulse duration and fall to zero away from the center of the pulse (at $t=0$ ). We shall assume that the phase upon transmission does not change, though in reality the random phase of magnetron transmitters is subsequently compensated for (coherent on receive). For simplicity, the target is assumed to be stationary and located on the same horizontal plane as the radar beam axis. If the pulse is reflected by a point target at range $\left(r_{\text {targ }}\right)$, the received waveform is a delayed replica of the original with an additional $180^{\circ}$ phase shift from reflection. The delay depends on the target range and the mean refractive index along the path $(n)$ :

$$
R(t) \propto e^{j\left\{2 \pi f_{\mathrm{TX}}\left[t-\left(2 r_{\operatorname{targ}} n / c\right)\right]+\pi\right\}}
$$

This waveform is mixed with a signal from the STALO (frequency $f_{\mathrm{ST}}$ ) and filtered to obtain an intermediate frequency, $\mathrm{IF}=f_{\mathrm{Tx}}-f_{\mathrm{ST}}$ :

$$
R_{\mathrm{IF}}(t) \propto e^{j\left\{2 \pi f_{\mathrm{TX}}\left[t-\left(2 r_{\mathrm{targ}} n / c\right)\right]+\pi\right\}} e^{-j 2 \pi f_{\mathrm{ST}} t} .
$$

This is mixed down to baseband using either a NCO (for digital IF) with frequency $\left(f_{\mathrm{NCO}}\right)$ or a $\mathrm{COHO}$ (for ana$\log$ IF systems). The baseband signal is expressed here in terms of the sum of the LO frequencies $\left(f_{\mathrm{LO}}=f_{\mathrm{ST}}+\right.$ $\left.f_{\mathrm{NCO}}\right)$ :

$$
\begin{aligned}
& R_{\mathrm{BB}}(t) \propto e^{j\left\{2 \pi f_{\mathrm{TX}}\left[t-\left(2 r_{\mathrm{targ}} n / c\right)\right]+\pi\right\}} e^{-j 2 \pi f_{\mathrm{ST}} t} e^{-j 2 \pi f_{\mathrm{NCO}} t} \\
& =e^{j\left\{2 \pi f_{\mathrm{TX}}\left[t-\left(2 r_{\mathrm{targ}} n / c\right)\right]+\pi\right\}} e^{-j 2 \pi f_{\mathrm{LO}} t}
\end{aligned}
$$

The sign convention used here for the phase of the transmitted and local oscillator frequencies is consistent with down conversion (i.e., positive frequency shifted to zero). The received waveform is sampled at a constant 
rate set by a master clock, which determines the rangegate separation. Let us consider the waveform at a time $\left(t_{\text {gate }}=2 r_{\text {gate }} / c\right)$ after transmission where $r_{\text {gate }}$ is the equivalent range-gate center in a vacuum (this definition is necessary to explicitly describe the phase dependence on refractivity changes). We shall now express this signal in terms of range rather than time to assist its interpretation:

$$
R_{\mathrm{BB}}\left(r_{\text {gate }}\right) \propto e^{j\left\{\left[4 \pi f_{\mathrm{Tx}}\left(r_{\text {gate }}-n r_{\text {targ }}\right) / c\right]+\pi\right\}} e^{-j\left(4 \pi f_{\mathrm{LO}} r_{\text {gate }} / c\right)} .
$$

The phase of the received signal from a particular target $(\operatorname{targ})$ is

$\phi_{\text {gate }}=\frac{4 \pi}{c}\left[f_{\text {Tx }}\left(r_{\text {gate }}-n r_{\text {targ }}\right)-f_{\text {LO }} r_{\text {gate }}\right]+\pi$.

Expressing this in terms of refractivity $\left[N=(n-1) 10^{6}\right]$ and target location relative to the range-gate center $\left(\delta_{\text {gate }}=r_{\text {targ }}-r_{\text {gate }}\right)$ gives after some rearrangement:

$$
\phi_{\text {gate }}=-\frac{4 \pi}{c}\left(f_{\mathrm{LO}} r_{\text {gate }}+f_{\mathrm{Tx}} \delta_{\text {gate }}+f_{\mathrm{Tx}} N 10^{-6} r_{\text {targ }}\right)+\pi .
$$

Let us now consider the phase change at some later time, where $f_{\mathrm{Tx}}, f_{\mathrm{LO}}$, and $n$ may all have changed; the subscripts 1 and 2 relate to the earlier and later measurements, respectively:

$$
\begin{aligned}
\Delta \phi_{\text {gate }}= & -\frac{4 \pi}{c}\left[\Delta f_{\mathrm{LO}} r_{\text {gate }}+\Delta f_{\mathrm{Tx}} \delta_{\text {gate }}\right. \\
& \left.+\left(f_{\mathrm{Tx}_{2}} N_{2}-f_{\mathrm{Tx}_{1}} N_{1}\right) 10^{-6} r_{\text {targ }}\right] .
\end{aligned}
$$

As $N_{2}=N_{1}+\Delta N$, it follows that $f_{\mathrm{Tx}_{2}} N_{2}-f_{\mathrm{Tx}_{1}} N_{1}=$ $f_{\mathrm{Tx}_{2}} \Delta N+\Delta f_{\mathrm{Tx}_{\mathrm{x}}} N_{1}$. Having made no approximations up to this point, the phase change for a stationary target may then be expressed as

$$
\begin{aligned}
\Delta \phi_{\text {gate }}= & -\frac{4 \pi}{c}\left[\Delta f_{\mathrm{LO}} r_{\text {gate }}+\Delta f_{\mathrm{Tx}} \delta_{\text {gate }}\right. \\
& \left.+\left(f_{\mathrm{Tx}_{2}} \Delta N+\Delta f_{\mathrm{Tx}} N_{1}\right) 10^{-6} r_{\text {targ }}\right]
\end{aligned}
$$

If we now assume a maximum fractional frequency change of $100 \mathrm{ppm}$ [e.g., $560 \mathrm{kHz}$ at $\mathrm{C}$ band $(5.6 \mathrm{GHz})]$, the second term in parentheses in (A9) will be negligible if $\Delta N \gg N_{1} 10^{-4}$. As near-surface refractivity values are typically within the range from 250 to 400 , the error in $\Delta N$ from this assumption would be less than 0.04 and may be neglected. Finally, considering that the frequency changes are small, we use the approximation
$f_{\mathrm{Tx}_{2}} \approx f_{\mathrm{Tx}}$ (i.e., $\Delta f_{\mathrm{Tx}} \ll f_{\mathrm{Tx}}$ ) and substituting $\Delta n=$ $\Delta N 10^{-6}$, we finally have

$$
\begin{aligned}
\Delta \phi_{\text {gate }}= & -\frac{4 \pi}{c}\left[\Delta f_{\mathrm{LO}} r_{\text {gate }}+\Delta f_{\mathrm{Tx}} \delta_{\text {gate }}\right. \\
& \left.+f_{\mathrm{Tx}} \Delta n\left(r_{\text {gate }}+\delta_{\text {gate }}\right)\right] .
\end{aligned}
$$

\section{REFERENCES}

Bartholomew, K., 2012: Assessing the potential of radar refractivity retrievals for improved high resolution weather prediction. Ph.D. thesis, University of Reading, Reading, United Kingdom, 178 pp.

Bean, B. R., and E. J. Dutton, 1968: Radio Meteorology. Dover Publications, $435 \mathrm{pp}$.

Bodine, D., and Coauthors, 2011: Understanding radar refractivity: Sources of uncertainty. J. Appl. Meteor. Climatol., 50, 25432560.

Cheong, B. L., R. D. Palmer, C. D. Curtis, T.-Y. Yu, D. S. Zrnic, and D. Forsyth, 2008: Refractivity retrieval using a phased array radar: First results and potential for multi-function operation. IEEE Trans. Geosci. Remote Sens., 46, 2527-2537.

Darlington, T., cited 2010: Weather radar signal processing. U.S. patent number 20100052976. [Available online at http://www. freepatentsonline.com/y2010/0052976.html.]

Doviak, R. J., and D. S. Zrnic, 2006: Doppler Radar and Weather Observations. 2nd ed. Dover Publications, 562 pp.

Fabry, F., 2004: Meteorological value of ground target measurements by radar. J. Atmos. Oceanic Technol., 21, 560-573.

- 2006: The spatial variability of moisture in the boundary layer and its effect on convective initiation: Project-long characterization. Mon. Wea. Rev., 134, 79-91.

— C. Frush, I. Zawadzki, and A. Kilambi, 1997: On the extraction of near-surface index of refraction using radar phase measurements from ground targets. J. Atmos. Oceanic Technol., 14, 978-987.

Hubbert, J. C., M. Dixon, S. M. Ellis, and G. Meymaris, 2009: Weather radar ground clutter. Part I: Identification, modeling, and simulation. J. Atmos. Oceanic Technol., 26, 1165-1180.

Junyent, F., V. Chandrasekar, and N. Bharadwaj, 2009: Uncertainties in phase and frequency estimation with a magnetron radar: Implication for clear air measurements. Proc. IEEE Int. Geoscience and Remote Sensing Symp. 2009, Cape Town, South Africa, IEEE, doi:10.1109/IGARSS. 2009.5417832.

,$- \ldots$, D. McLaughlin, E. Insanic, and N. Bharadwaj, 2010: The CASA Integrated Project 1 networked radar system. J. Atmos. Oceanic Technol., 27, 61-78.

Kudeki, E., and G. R. Stitt, 1987: Frequency domain interferometry: A high-resolution radar technique for studies of atmospheric turbulence. Geophys. Res. Lett., 14, 198-201.

Nicol, J. C., and A. J. Illingworth, 2013: The effect of phase-correlated returns and spatial smoothing on the accuracy of radar refractivity retrievals. J. Atmos. Oceanic Technol., 30, 22-39.

, T. Darlington, A. Illingworth, K. Bartholomew, M. Kitchen, and E. Delaygue, 2008: Operational testing of radar refractivity retrieval for the UK radar network. Extended $A b$ stracts, Fifth European Conf. on Radar Meteorology and Hydrology, Helsinki, Finland, FMI, CD-ROM.

, K. Bartholomew, T. Darlington, A. Illingworth, and M. Kitchen, 2012: Operational radar refractivity retrieval for 
numerical weather prediction. Weather Radar and Hydrology, R. J. Moore, S. J. Cole, and A. J. Illingworth, Eds., IAHS Publ. 351, 348-353.

, A. J. Illingworth, and K. Bartholomew, 2013: The potential of one-hour refractivity changes from an operational C-band magnetron-based radar for NWP validation and data assimilation. Quart. J. Roy. Meteor. Soc., doi:10.1002/qj.2223, in press.

Parent du Chatelet, J., and C. Boudjabi, 2008: A new formulation for signal reflected from a target using a magnetron radar: Consequences for Doppler and refractivity measurements. Extended Abstracts, Fifth European Conf. on Radar Meteorology and Hydrology, Helsinki, Finland, FMI, CD-ROM.

-, P. Tabary, and C. Boudjabi, 2007: Evaluation of the refractivity measurement feasibility with a C-band radar equipped with a magnetron transmitter. Extended Abstracts, 33rd Int. Conf. on Radar Meteorology, Cairns, Australia, Amer. Meteor. Soc., 8B.6. [Available online at https://ams. confex.com/ams/33Radar/webprogram/Paper123581.html.]
— C. Boudjabi, L. Besson, and O. Caumont, 2012: Errors caused by long-term drifts of magnetron frequencies for refractivity measurement with a radar: Theoretical formulation and initial validation. J. Atmos. Oceanic Technol., 29, 1428-1434.

Park, S., and F. Fabry, 2010: Simulation and interpretation of the phase data used by the radar refractivity retrieval algorithm. J. Atmos. Oceanic Technol., 27, 1286-1301.

Roberts, R. D., and Coauthors, 2008: REFRACTT-2006: Realtime retrieval of high-resolution, low-level moisture fields from operational NEXRAD and research radars. Bull. Amer. Meteor. Soc., 89, 1535-1548.

Skolnik, M., 1990: Radar Handbook. 2nd ed. McGraw-Hill, 1200 pp. Weber, R., 1997: Estimators for the standard deviation of horizontal wind direction. J. Appl. Meteor., 36, 1403-1415.

Weckwerth, T. M., C. R. Pettet, F. Fabry, S. Park, M. A. LeMone, and J. W. Wilson, 2005: Radar refractivity retrieval: Validation and application to short-term forecasting. J. Appl. Meteor., 44, 285-300. 